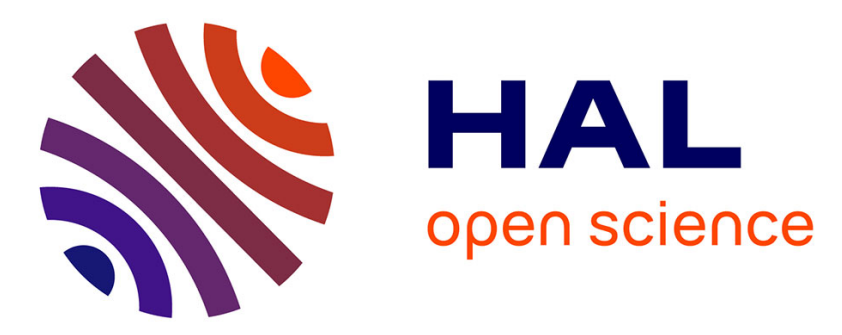

\title{
Edge Stress Intensity Functions in Polyhedral Domains and their Extraction by a Quasidual Function Method
}

Zohar Yosibash, Netta Omer, Martin Costabel, Monique Dauge

\section{To cite this version:}

Zohar Yosibash, Netta Omer, Martin Costabel, Monique Dauge. Edge Stress Intensity Functions in Polyhedral Domains and their Extraction by a Quasidual Function Method. International Journal of Fracture, 2005, 136 (1-4), pp.37-73. hal-00012184

\section{HAL Id: hal-00012184 https://hal.science/hal-00012184}

Submitted on 17 Oct 2005

HAL is a multi-disciplinary open access archive for the deposit and dissemination of scientific research documents, whether they are published or not. The documents may come from teaching and research institutions in France or abroad, or from public or private research centers.
L'archive ouverte pluridisciplinaire HAL, est destinée au dépôt et à la diffusion de documents scientifiques de niveau recherche, publiés ou non, émanant des établissements d'enseignement et de recherche français ou étrangers, des laboratoires publics ou privés. 


\title{
Edge Stress Intensity Functions in Polyhedral Domains and their Extraction by a Quasidual Function Method
}

\author{
Zohar Yosibash $^{1}$, Netta Omer ${ }^{1},{\text { Martin } \text { Costabel }^{2} \text { and Monique Dauge }}^{2}$ \\ 1 Dept. of Mechanical Engineering., Ben-Gurion University of the Negev, Beer-Sheva, 84105, ISRAEL, \\ 2 UMR CNRS 6625 - IRMAR, Université de Rennes 1 - Campus de Beaulieu, 35042 Rennes Cedex, FRANCE
}

\begin{abstract}
The solution to elastic isotropic problems in three-dimensional (3-D) polyhedral domains in the vicinity of an edge is provided in an explicit form. It involves a family of eigen-functions with their shadows, and the associated edge stress intensity functions (ESIFs), which are functions along the edges. Utilizing the explicit structure of the solution in the vicinity of the edge we use the quasidual function method, recently presented in [12] for scalar elliptic problems and in [5] in a general framework, for the extraction of ESIFs.

This accurate and efficient method provides a polynomial approximation of the ESIF along the edge whose order is adaptively increased so to approximate the exact ESIF. It is implemented as a post-solution operation in conjunction with the $p$-version finite element method. Numerical examples are provided in which we extract ESIFs associated with traction free or homogeneous Dirichlet boundary conditions in 3-D cracked domains or 3-D V-Notched domains. These demonstrate the efficiency, robustness and high accuracy of the proposed quasi-dual function method.
\end{abstract}

Keywords: J-integral, edge stress intensity functions, high order finite elements

\section{Introduction}

Solutions to linear elastic problems in two-dimensional (2-D) polygonal domains in the vicinity of reentrant corners, and especially crack tips, have been studied for over half a decade and known to be expressed as an asymptotic series. These are described in terms of special singular functions (eigen-functions) depending on the geometry and the boundary conditions in the vicinity of the corner on one hand, and of unknown coefficients (stress intensity factors) depending on the given body forces and tractions on the other hand. The eigen-pairs (eigen-values and eigenfunctions) may be obtained by several techniques. An analytical method for computing eigen-pairs in isotropic domains is provided in many prior publications $[14,10,7,3]$. A semi-analytic approach for the eigen-pairs computations was presented in [4], applicable to anisotropic domains. Many numerical methods were developed, as for example in [11], [13], and [15] which are applicable also to anisotropic and multi-material interfaces.

In three-dimensional polyhedron domains, however, the solution is represented by three different asymptotic expansions based on it's vicinity to either an edge, a vertex or a vertex-edge [6]. Herein we concentrate our attention to the solution in the vicinity of edges. It's representation is characterized:

(C) 2005 Kluwer Academic Publishers. Printed in the Netherlands.

EI_ESIF.tex; 26/04/2005; $17: 44 ;$ p.1 
- by an exponent $\alpha$ which belongs to a discrete set $\left\{\alpha_{i}, i \in \mathbb{N}\right\}$ of eigen-values depending only on the geometry, material properties and boundary conditions in the vicinity of the singularity, and which determines the level of non-smoothness of the singularity. Any eigenvalue $\alpha_{i}$ is computed by solving a $2-\mathrm{D}$ problem.

- by an associated eigen-function $\varphi_{0}^{(\alpha)}(\theta)$ which depends on the geometry of the domain and material properties. These eigen-functions are computed by solving a set of 2 -D problems.

- by a function along the edge, denoted by $A_{i}\left(x_{3}\right)$ ( $x_{3}$ is a coordinate along the edge). $A_{i}\left(x_{3}\right)$ is associated with the $i^{\text {th }}$ eigen-value and called "Edge Stress Intensity Function" (ESIF) which determines the "amount of energy" residing in each singularity.

From the engineering perspective the ESIFs $A_{i}\left(x_{3}\right)$ when $\alpha_{i}<1$ are of major importance because these are correlated to failure initiation. In many situations $\alpha_{i}<1$ when the opening at the edge is non-convex. For example $\alpha_{i}$ can be equal to $\frac{1}{2}$ in the presence of cracks.

The aim of this paper is two-fold - first to provide the mathematical algorithm for the construction of the asymptotic elastic solution in the vicinity of an edge (which is an extension of the two-dimensional case), and second, to compute a polynomial approximation of the edge stress intensity function by a new extraction method explained in details in [12] on the basis of the scalar elliptic problem.

The eigen-pairs of the three dimensional cracked or notched domain in the vicinity of the edge were first addressed by Hartranft and Sih in [8], shown to be computed by a recursive procedure. At the time however, these were not presented explicitly and the general structure of the asymptotic expansion not observed.

Herein, the abstract formulation in [5], and its applicability to scalar elliptic problem reported in [12] are used to explicitly express the elasticity solution in the vicinity of an edge as a combination of eigen-functions and their shadows. These shadows are "new functions" appearing in 3-D domains, having no counterparts in 2-D domains as far as homogeneous operators with constant coefficients are concerned. The dual eigen-functions and their dual shadows are computed also, which are required subsequently for the quasi-dual function method. Using the eigen-functions and their shadows, the functional $J[R]$ is used (following $[5,12]$ ), which can be viewed as an extension of the 2-D contour integral to 3 -D domains. This new functional, which is a surface integral along a cylindrical surface, enables us to present the edge stress intensity function explicitly as a function of $x_{3}$ (the coordinate along the edge). The method presented is implemented as a post-processing step in a $p$-version finite element code and the numerical performance is documented on several example problems. By the $J[R]$ functional, and newly constructed extraction polynomials, we extract the ESIFs in the vicinity of any edge (including crack front) in any polyhedron. This method provides the functional representation of the ESIFs along $x_{3}$ (as opposed to other methods providing point-wise values of the ESIFs along the edge) and is very accurate, efficient and robust. Most importantly, the method is adaptive, providing a better polynomial representation of the ESIF as the special hierarchical family of extraction polynomials is increased. We extract 
the ESIF for several problems for which an analytical solution exists to demonstrate its accuracy and efficiency.

This paper is organized as follows:

- We start with notations, defining the domain of interest and the linear elastic problem.

- The mathematical algorithm is then presented for obtaining the asymptotic expansion of the solution in the neighborhood of an edge in terms of eigen-functions, their shadows, and the structure of the ESIFs. The dual eigen-functions, and their shadows, which are associated with the primal eigen-functions are addressed as well.

- The $J[R]$ integral is then introduced [5]. It requires the construction of extracting polynomials, denoted by $B\left(x_{3}\right)$, and the data on a cylindrical surface of radius $R$ around the edge. A short explanation on its application in conjunction with the finite element method is given.

- Subsequently, a hierarchical family of extraction polynomials is constructed.

- The hierarchical family of extraction polynomials is used in several numerical tests to extract the ESIFs associated with:

- A cracked domain with traction free boundary conditions.

- V-notched domain with clamped boundary conditions.

For these two problems we provide in Appendices A and B the explicit formulas for the eigenfunctions, duals and shadows. Numerical experimentations are performed to demonstrate that the polynomial representation of the ESIF for both example problem is accurate and efficient.

- Finally, we present an example problem of engineering relevance. We examine a compact test specimen subjected to tension load such that only Mode $I$ is excited along the crack front. We compare the extracted ESIF by our method with a point-wise extraction method. This example problem demonstrates the efficiency and robustness of the quasi-dual function method in handling realistic geometries in engineering practice.

\section{The elastic solution for an isotropic problem in the vicinity of an edge.}

In this section we derive the asymptotic solution in the neighborhood of an edge in an isotropic elastic domain. It is shown that the elastic solution can be presented as an asymptotic series of eigen-pairs (the well known eigen-pairs of the 2-D cross section) and the associated edge stress intensity functions. However, as opposed to planar elastic problems, each of the eigen-pairs is accompanied by an infinite number of shadow functions with an increasing exponential order. 


\subsection{Differential Equations for the 3-D Eigen-Pairs}

Consider a domain $\Omega$ in which one straight edge $\mathcal{E}$ of interest is present. The domain is generated as the product $\Omega=G \times I$ where $I$ is the interval $[-1,1]$, and $G$ is a plane bounded sector of opening $\omega \in(0,2 \pi]$ and for simplicity assume it has a radius 1 (the case of a crack, $\omega=2 \pi$, is included), as shown in Figure 1. Although any $G$ or $I$ can be chosen, these simplified ones have been chosen for simplicity of presentation.

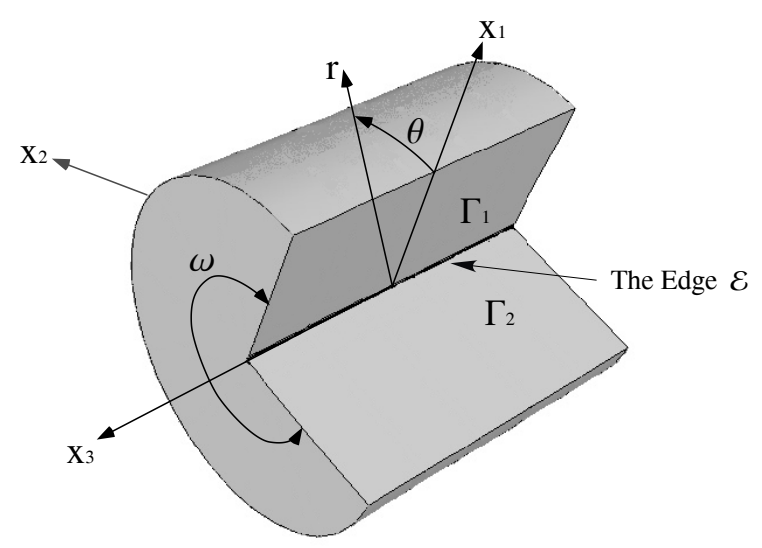

Figure 1. Domain of interest $\Omega$.

The variables in $G$ and $I$ are $\left(x_{1}, x_{2}\right)$ and $x_{3}$ respectively, and the coordinates $\left(x_{1}, x_{2}, x_{3}\right)$ are denoted by $\boldsymbol{x}$. Let $(r, \theta)$ be the polar coordinates centered at the vertex of $G$ so that $G$ coincides with $\left\{\left(x_{1}, x_{2}\right) \in \mathbb{R}^{2} \mid r \in(0,1), \theta \in(0, \omega)\right\}$. The edge $\mathcal{E}$ of interest is the set $\left\{\boldsymbol{x} \in \mathbb{R}^{3} \mid r=0, x_{3} \in I\right\}$. The two flat planes that intersect at the edge $\mathcal{E}$ are denoted by $\Gamma_{1}$ and $\Gamma_{2}$. For any $R, 0<R<1$, the cylindrical surface $\Gamma_{R}$ is defined as follows:

$$
\Gamma_{R}:=\left\{\boldsymbol{x} \in \mathbb{R}^{3} \mid r=R, \theta \in(0, \omega), x_{3} \in I\right\}
$$

REMARK 1. The methods presented in the paper are restricted to geometries where the edges are straight lines and the angle $\omega$ is fixed along $x_{3}$.

REMARK 2. In general the eigen-pairs associated with the elasticity operator may be complex, however in most practical cases the eigen-values smaller than 1 are of interest, and these are usually real. Herein we address real eigen-pairs only, whereas the general case will be addressed in a future publication.

To distinguish between the displacements vector in Cartesian or Polar coordinates, we denote these by $\boldsymbol{u}=\left\{u_{1}, u_{2}, u_{3}\right\}^{T}, \tilde{\boldsymbol{u}}=\left\{u_{r}, u_{\theta}, u_{x_{3}}\right\}^{T}$ respectively and use either of them when convenient. 
The Navier-Lamé equations that describe the elastic isotropic problem in polar coordinates is:

$$
\begin{gathered}
(\lambda+2 \mu) \partial_{r}^{2} u_{r}+(\lambda+2 \mu) \frac{1}{r} \partial_{r} u_{r}-(\lambda+2 \mu) \frac{1}{r^{2}} u_{r}+\mu \frac{1}{r^{2}} \partial_{\theta}^{2} u_{r}+\mu \partial_{3}^{2} u_{r} \\
-(\lambda+3 \mu) \frac{1}{r^{2}} \partial_{\theta} u_{\theta}+(\lambda+\mu) \frac{1}{r} \partial_{r} \partial_{\theta} u_{\theta}+(\lambda+\mu) \partial_{r} \partial_{3} u_{3}=0 \\
(\lambda+\mu) \frac{1}{r} \partial_{r} \partial_{\theta} u_{r}+(\lambda+3 \mu) \frac{1}{r^{2}} \partial_{\theta} u_{r}+(\lambda+2 \mu) \frac{1}{r^{2}} \partial_{\theta}^{2} u_{\theta}+\mu \partial_{r}^{2} u_{\theta} \\
+\mu \frac{1}{r} \partial_{r} u_{\theta}-\mu \frac{1}{r^{2}} u_{\theta}+\mu \partial_{3}^{2} u_{\theta}+(\lambda+\mu) \frac{1}{r} \partial_{3} \partial_{\theta} u_{3}=0 \\
(\lambda+\mu) \partial_{r} \partial_{3} u_{r}+(\lambda+\mu) \frac{1}{r} \partial_{3} u_{r}+(\lambda+\mu) \frac{1}{r} \partial_{3} \partial_{\theta} u_{\theta}+\mu \partial_{r}^{2} u_{3} \\
+\mu \frac{1}{r} \partial_{r} u_{3}+\mu \frac{1}{r^{2}} \partial_{\theta}^{2} u_{3}+(\lambda+2 \mu) \partial_{3}^{2} u_{3}=0
\end{gathered}
$$

with $\lambda, \mu$ being the Lamé constants associated with the engineering material constants $E$ the Young modulus and $\nu$ the Poisson ratio. The system (2)-(4) can be splitted into three operators:

$$
\mathcal{L}(\tilde{\boldsymbol{u}})=\left[M_{0}\left(\partial_{r}, \partial_{\theta}\right)\right] \tilde{\boldsymbol{u}}+\left[M_{1}\left(\partial_{r}, \partial_{\theta}\right)\right] \partial_{3} \tilde{\boldsymbol{u}}+\left[M_{2}\left(\partial_{r}, \partial_{\theta}\right)\right] \partial_{3}^{2} \tilde{\boldsymbol{u}}=0
$$

with:

$$
\begin{gathered}
{\left[M_{0}\right]=\left(\begin{array}{ccc}
(\lambda+2 \mu)\left(\partial_{r}^{2}+\frac{1}{r} \partial_{r}-\frac{1}{r^{2}}\right)+\mu \frac{1}{r^{2}} \partial_{\theta}^{2} & -(\lambda+3 \mu) \frac{1}{r^{2}} \partial_{\theta}+(\lambda+\mu) \frac{1}{r} \partial_{r} \partial_{\theta} & 0 \\
(\lambda+\mu) \frac{1}{r} \partial_{r} \partial_{\theta}+(\lambda+3 \mu) \frac{1}{r^{2}} \partial_{\theta} & (\lambda+2 \mu) \frac{1}{r^{2}} \partial_{\theta}^{2}+\mu\left(\partial_{r}^{2}+\frac{1}{r} \partial_{r}-\frac{1}{r^{2}}\right) & 0 \\
0 & 0 & \mu\left(\partial_{r}^{2}+\frac{1}{r} \partial_{r}+\frac{1}{r^{2}} \partial_{\theta}^{2}\right)
\end{array}\right)} \\
{\left[M_{1}\right]=\left(\begin{array}{ccc}
0 & 0 & (\lambda+\mu) \partial_{r} \\
0 & 0 & (\lambda+\mu) \frac{1}{r} \partial_{\theta} \\
(\lambda+\mu)\left(\partial_{r}+\frac{1}{r}\right) & (\lambda+\mu) \frac{1}{r} \partial_{\theta} & 0
\end{array}\right), \quad\left[M_{2}\right]=\left(\begin{array}{ccc}
\mu & 0 & 0 \\
0 & \mu & 0 \\
0 & 0 & (\lambda+2 \mu)
\end{array}\right)}
\end{gathered}
$$

The splitting allows the consideration of a solution $\tilde{\boldsymbol{u}}$ of the form:

$$
\tilde{\boldsymbol{u}}=\sum_{j \geq 0} \partial_{3}^{j} A\left(x_{3}\right) \mathbf{\Phi}_{j}(r, \theta)
$$

The N-L system in view of (8) becomes:

$$
\sum_{j \geq 0} \partial_{3}^{j} A\left(x_{3}\right)\left[M_{0}\right] \mathbf{\Phi}_{j}+\sum_{j \geq 0} \partial_{3}^{j+1} A\left(x_{3}\right)\left[M_{1}\right] \mathbf{\Phi}_{j}+\sum_{j \geq 0} \partial_{3}^{j+2} A\left(x_{3}\right)\left[M_{2}\right] \boldsymbol{\Phi}_{j}=0
$$

and after rearranging:

$$
\begin{gathered}
A\left(x_{3}\right)\left[M_{0}\right] \boldsymbol{\Phi}_{0}+\partial_{3} A\left(x_{3}\right)\left(\left[M_{0}\right] \mathbf{\Phi}_{1}+\left[M_{1}\right] \mathbf{\Phi}_{0}\right)+ \\
+\sum_{j \geq 0} \partial_{3}^{j+2} A\left(x_{3}\right)\left(\left[M_{0}\right] \mathbf{\Phi}_{j+2}+\left[M_{1}\right] \boldsymbol{\Phi}_{j+1}+\left[M_{2}\right] \mathbf{\Phi}_{j}\right)=0
\end{gathered}
$$

Equation (10) has to hold for any smooth function $A\left(x_{3}\right)$. Thus, the functions $\boldsymbol{\Phi}_{j}$ must satisfy the three equations below, each defined on a two-dimensional domain $G$ :

$$
\left\{\begin{array}{l}
{\left[M_{0}\right] \boldsymbol{\Phi}_{0}=0} \\
{\left[M_{0}\right] \boldsymbol{\Phi}_{1}+\left[M_{1}\right] \boldsymbol{\Phi}_{0}=0} \\
{\left[M_{0}\right] \boldsymbol{\Phi}_{j+2}+\left[M_{1}\right] \boldsymbol{\Phi}_{j+1}+\left[M_{2}\right] \boldsymbol{\Phi}_{j}=0, \quad j \geq 0}
\end{array} \quad(r, \theta) \in G\right.
$$


accompanied by homogeneous boundary conditions on the two surfaces $\Gamma_{1}$ and $\Gamma_{2}$, discussed in the sequel.

The first partial differential equation in (11) generates the solution $\boldsymbol{\Phi}_{0}$ associated with the eigen-value $\alpha$, denoted primal singular function, which is the well known two-dimensional eigenfunction:

$$
\boldsymbol{\Phi}_{0}=r^{\alpha} \boldsymbol{\varphi}_{0}(\theta)
$$

The second PDE in (11) generates the function $\boldsymbol{\Phi}_{1}$ which depends on $\boldsymbol{\Phi}_{0}$ :

$$
\boldsymbol{\Phi}_{1}=r^{\alpha+1} \boldsymbol{\varphi}_{1}(\theta)
$$

The sequence $\boldsymbol{\Phi}_{j}$ (where $j \geq 2$ ) are the solutions of the third equation of (11). These are of the form:

$$
\boldsymbol{\Phi}_{j}=r^{\alpha+j} \boldsymbol{\varphi}_{j}(\theta)
$$

All $\boldsymbol{\Phi}_{j}, j>1$ are called shadow functions of the primal singular function $\boldsymbol{\Phi}_{0}$. There exist an infinite number of shadow functions $\boldsymbol{\Phi}_{j}$ for each eigen-value $\alpha_{i}$ (these are obtained by applying boundary conditions as will be discussed in subsection 2.2):

$$
\boldsymbol{\Phi}_{j}^{\left(\alpha_{i}\right)}=r^{\alpha_{i}+j} \boldsymbol{\varphi}_{j}^{\left(\alpha_{i}\right)}(\theta) \quad j=0,1, \cdots
$$

Thus, for each eigen-value $\alpha_{i}$, the 3 -D solution, in the vicinity of an edge is:

$$
\tilde{\boldsymbol{u}}^{\left(\alpha_{i}\right)}=\sum_{j \geq 0} \partial_{3}^{j} A_{i}\left(x_{3}\right) r^{\alpha_{i}+j} \boldsymbol{\varphi}_{j}^{\left(\alpha_{i}\right)}(\theta)
$$

and the overall solution $\tilde{\boldsymbol{u}}$ is:

$$
\tilde{\boldsymbol{u}}=\sum_{i \geq 1} \tilde{\boldsymbol{u}}^{\left(\alpha_{i}\right)}=\sum_{i \geq 1} \sum_{j \geq 0} \partial_{3}^{j} A_{i}\left(x_{3}\right) r^{\alpha_{i}+j} \boldsymbol{\varphi}_{j}^{\left(\alpha_{i}\right)}(\theta)
$$

where $A_{i}\left(x_{3}\right)$ is the Edge Stress Intensity Function (ESIF) of the $i^{\text {th }}$ eigen-value.

Solutions of (11) for the negative eigen-values are called the dual singular solutions, and are denoted by $\boldsymbol{\Psi}$. Because the operator $\mathcal{L}$ is self-adjoint, for any $\alpha_{i}$ the number $-\alpha_{i}$ is also an eigen-value and there exists $\Psi_{0}^{\left(\alpha_{i}\right)}$ such that $r^{-\alpha_{i}} \mathbf{\Psi}_{0}^{\left(\alpha_{i}\right)}(\theta)$ solves the first equation of (11). For normalization purpose a real coefficient $c_{0}^{\left(\alpha_{i}\right)}$ is chosen:

$$
\mathbf{\Psi}_{0}^{\left(\alpha_{i}\right)}=c_{0}^{\left(\alpha_{i}\right)} r^{-\alpha_{i}} \boldsymbol{\psi}_{0}^{\left(\alpha_{i}\right)}(\theta)=c_{0}^{\left(\alpha_{i}\right)} r^{-\alpha_{i}} \boldsymbol{\varphi}_{0}^{\left(-\alpha_{i}\right)}(\theta)
$$

where $\Psi_{0}^{\left(\alpha_{i}\right)}$ is the dual leading eigen-solution and

$$
\boldsymbol{\Psi}_{j}^{\left(\alpha_{i}\right)}=c_{0}^{\left(\alpha_{i}\right)} r^{-\alpha_{i}+j} \boldsymbol{\psi}_{j}^{\left(\alpha_{i}\right)}(\theta)=c_{0}^{\left(\alpha_{i}\right)} r^{-\alpha_{i}+j} \boldsymbol{\varphi}_{j}^{\left(-\alpha_{i}\right)}(\theta)
$$

are the shadow dual eigen-solutions. Theoretical details and rigorous mathematical formulation is provided in [5]. Detailed explanation about the shadow functions of the scalar elliptic problem is presented in [12]. 


\subsection{BOUNDARY CONDITIONS FOR THE PRIMAL AND DUAL SHADOW FUNCTIONS}

Two types of boundary conditions are considered on $\Gamma_{1}$ and $\Gamma_{2}$ surfaces, either traction free or clamped.

\subsubsection{Traction Free Boundary Conditions}

The traction free boundary conditions on $\Gamma_{1}, \Gamma_{2}$ result in:

$$
\left.[T](\tilde{\boldsymbol{u}})\right|_{\Gamma_{1}, \Gamma_{2}}=\left.\left(\left[T_{0}\left(\partial_{r}, \partial_{\theta}\right)\right] \tilde{\boldsymbol{u}}+\left[T_{1}\left(\partial_{r}, \partial_{\theta}\right)\right] \partial_{3} \tilde{\boldsymbol{u}}\right)\right|_{\Gamma_{1}, \Gamma_{2}}=0
$$

Inserting (8) in (20) one obtains:

$$
\left.A\left(x_{3}\right)\left[T_{0}\right] \mathbf{\Phi}_{0}\right|_{\Gamma_{1}, \Gamma_{2}}+\left.\sum_{j \geq 0} \partial_{3}^{j+1} A\left(x_{3}\right)\left(\left[T_{0}\right] \boldsymbol{\Phi}_{j+1}+\left[T_{1}\right] \boldsymbol{\Phi}_{j}\right)\right|_{\Gamma_{1}, \Gamma_{2}}=0
$$

Equation (21) has to hold for any smooth function $A\left(x_{3}\right)$ and therefore the boundary conditions for the eigen-functions are:

$$
\left\{\begin{array}{l}
{\left[T_{0}\right] \mathbf{\Phi}_{0}=0} \\
{\left[T_{0}\right] \mathbf{\Phi}_{j+1}+\left[T_{1}\right] \boldsymbol{\Phi}_{j}=0, \quad j \geq 0}
\end{array} \quad \text { on } \Gamma_{1}, \Gamma_{2}\right.
$$

The first equation in (22) is the boundary conditions for $\boldsymbol{\Phi}_{0}$ which is identical to the twodimensional boundary conditions. The second equation in (22) is the boundary conditions for each $\boldsymbol{\Phi}_{j}$ where $j \geq 1$.

The operator-matrices $\left[T_{0}\right]$ and $\left[T_{1}\right]$ are explicitly obtained if traction free boundary conditions (20) are considered on $\Gamma_{1}, \Gamma_{2}$ :

$$
\left\{\begin{array} { l } 
{ ( \sigma _ { r \theta } ) | _ { \theta = 0 , \omega } = 0 } \\
{ ( \sigma _ { \theta \theta } ) | _ { \theta = 0 , \omega } = 0 } \\
{ ( \sigma _ { \theta 3 } ) | _ { \theta = 0 , \omega } = 0 }
\end{array} \Rightarrow \left\{\begin{array}{l}
\left.\left(\mu\left(\frac{1}{r} \partial_{\theta} u_{r}+\partial_{r} u_{\theta}-\frac{1}{r} u_{\theta}\right)\right)\right|_{\theta=0, \omega}=0 \\
\left.\left((\lambda+2 \mu) \frac{1}{r} u_{r}+\lambda \partial_{r} u_{r}+(\lambda+2 \mu) \frac{1}{r} \partial_{\theta} u_{\theta}+\lambda \partial_{3} u_{3}\right)\right|_{\theta=0, \omega}=0 \\
\left.\left(\mu\left(\partial_{3} u_{\theta}+\frac{1}{r} \partial_{\theta} u_{3}\right)\right)\right|_{\theta=0, \omega}=0
\end{array}\right.\right.
$$

obtaining:

$$
\left[T_{0}\right]=\left(\begin{array}{ccc}
\mu \frac{1}{r} \partial_{\theta} & \mu \partial_{r}-\mu \frac{1}{r} & 0 \\
(\lambda+2 \mu) \frac{1}{r}+\lambda \partial_{r} & (\lambda+2 \mu) \frac{1}{r} \partial_{\theta} & 0 \\
0 & 0 & \mu \frac{1}{r} \partial_{\theta}
\end{array}\right), \quad\left[T_{1}\right]=\left(\begin{array}{ccc}
0 & 0 & 0 \\
0 & 0 & \lambda \\
0 & \mu & 0
\end{array}\right)
$$

\subsubsection{Clamped Boundary Conditions}

Clamped boundary conditions on $\Gamma_{1}, \Gamma_{2}$ are:

$$
\left.\tilde{\boldsymbol{u}}\right|_{\Gamma_{1}, \Gamma_{2}}=\left.\sum_{j \geq 0} \partial_{3}^{j} A\left(x_{3}\right) \boldsymbol{\Phi}_{j}(r, \theta)\right|_{\Gamma_{1}, \Gamma_{2}}=0
$$


Equation (24) has to hold for any smooth function $A\left(x_{3}\right)$ and therefore the clamped boundary conditions for the eigen-functions are:

$$
\boldsymbol{\Phi}_{j}(r, \theta)=0 \quad \text { on } \quad \Gamma_{1}, \Gamma_{2}
$$

Explicit expressions for the primal and dual eigen-functions and their shadows for a traction free

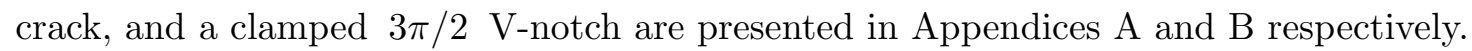

\section{Extracting ESIFs by the $J[R]$-integral}

Once the asymptotic series representing the elastic solution in the vicinity of an edge is available, we proceed to extraction of edge stress intensity functions by recalling the $J[R]$-integral introduced in [5]. The numerical performance of this extraction method has been presented for scalar elliptic problems in [12]. Herein we show an improvement of the method and apply it to the elasticity equations.

We start by constructing the quasidual-singular functions: for each eigen-value $\alpha_{i}$, a set of quasidual-singular functions $\mathbf{K}_{m}^{\left(\alpha_{i}\right)}[B]$ are constructed where $m$ is a natural integer called the order of the quasidual function, and $B\left(x_{3}\right)$ is a function (we choose it to be related to the Jacobi polynomials) called extraction polynomial. Each $\mathbf{K}_{m}^{\left(\alpha_{i}\right)}[B]$ is characterized by the number of dual singular functions $m$ needed to construct it and the extraction polynomial $B$ :

$$
\mathbf{K}_{m}^{\left(\alpha_{i}\right)}[B] \stackrel{\text { def }}{=} \sum_{j=0}^{m} \partial_{3}^{j} B\left(x_{3}\right) \mathbf{\Psi}_{j}^{\left(\alpha_{i}\right)} .
$$

By using the quasidual functions, one can extract a scalar product of $A_{i}\left(x_{3}\right)$ with $B\left(x_{3}\right)$ on $\mathcal{E}$. This is accomplished with the help of the anti-symmetric boundary integral $J[R]$, over the surface $\Gamma_{R}(1)$. We define $J[R](\mathbf{f}, \mathbf{v})$ to be:

$$
J[R](\mathbf{f}, \mathbf{v}) \stackrel{\text { def }}{=} \int_{\Gamma_{R}}\left(\left.[T]\right|_{\Gamma_{R}} \mathbf{f} \cdot \mathbf{v}-\left.\mathbf{f} \cdot[T]\right|_{\Gamma_{R}} \mathbf{v}\right) d S=\left.\int_{I} \int_{0}^{\omega}\left(\left.[T]\right|_{\Gamma_{R}} \mathbf{f} \cdot \mathbf{v}-\left.\mathbf{f} \cdot[T]\right|_{\Gamma_{R}} \mathbf{v}\right)\right|_{r=R} R d \theta d x_{3}
$$

where $I \equiv \mathcal{E}$ (the edge) along $x_{3}$ axis (Figure 1 ) and $\left.[T]\right|_{\Gamma_{R}}$ is the radial Neumann trace operator related to the operator $\mathcal{L}$ on $\Gamma_{R}$ surface:

$$
\left.[T]\right|_{\Gamma_{R}} \tilde{\boldsymbol{u}} \stackrel{\text { def }}{=}\left(\begin{array}{c}
\sigma_{r r} \\
\sigma_{r \theta} \\
\sigma_{r 3}
\end{array}\right)=\left(\begin{array}{ccc}
(\lambda+2 \mu) \partial_{r}+\lambda \frac{1}{r} & \lambda \frac{1}{r} \partial_{\theta} & \lambda \partial_{3} \\
\mu \frac{1}{r} \partial_{\theta} & -\mu \frac{1}{r}+\mu \partial_{r} & 0 \\
\mu \partial_{3} & 0 & \mu \partial_{r}
\end{array}\right)\left(\begin{array}{c}
u_{r} \\
u_{\theta} \\
u_{3}
\end{array}\right)
$$

With the above definitions we have the following theorem [5]:

THEOREM 1. Take $B\left(x_{3}\right)$ such that

$$
\partial_{3}^{j} B\left(x_{3}\right)=0 \quad \text { for } \quad j=0, \ldots, m-1 \quad \text { on } \partial I
$$


then, if the ESIFs $A_{i}$ in the expansion (17) are smooth enough:

$$
J[R]\left(\tilde{\boldsymbol{u}}, \mathbf{K}_{m}^{\left(\alpha_{i}\right)}[B]\right)=\int_{I} A_{i}\left(x_{3}\right) B\left(x_{3}\right) d x_{3}+\mathcal{O}\left(R^{\alpha_{1}-\alpha_{i}+m+1}\right), \quad \text { as } \quad R \rightarrow 0 .
$$

Here $\alpha_{1}$ is the smallest of the eigen-values $\alpha_{i}, i \in \mathbb{N}$.

Theorem 1 allows a precise determination of $\int_{I} A_{i}\left(x_{3}\right) B\left(x_{3}\right) d x_{3}$ by computing (30) for two or three $R$ values and using Richardson's extrapolation as $R \rightarrow 0$.

\subsection{Projection of the ESIFs into the space spanned By Jacobi Polynomials}

We are interested in extracting the $\operatorname{ESIF}\left(A_{i}\left(x_{3}\right)\right)$. Because its functional representation is unknown, its polynomial approximation is sought. According to Theorem 1 the computation of ESIFs is associated with a chosen extracting function $B\left(x_{3}\right)$ that has to satisfy several boundary conditions on $\partial I$. Choosing to represent $A_{i}\left(x_{3}\right)$ by a polynomial basis, we construct an adaptive class of orthonormal polynomials with a given weight $w\left(x_{3}\right)=\left(1-x_{3}^{2}\right)^{n}$ so to represent $B_{n}\left(x_{3}\right)$. This suggests the use of Jacobi polynomials as a natural basis. In this way, if $A_{i}\left(x_{3}\right)$ is a polynomial of degree $N$, it can be represented by a linear combination of Jacobi polynomials as:

$$
A_{i}\left(x_{3}\right)=\tilde{a}_{0} J_{n}^{(0)}+\tilde{a}_{1} J_{n}^{(1)}\left(x_{3}\right)+\cdots+\tilde{a}_{N} J_{n}^{(N)}\left(x_{3}\right)
$$

where $J_{n}^{(k)}$ is the Jacobi polynomial of degree $k$ and order $n$, i.e. associated with the weight $w\left(x_{3}\right)=\left(1-x_{3}^{2}\right)^{n}$, which is denoted in literature by $P_{k}^{(n, n)}$. There holds the following orthogonality property [2, pp. 773-774] :

$$
\int_{-1}^{1}\left(1-x_{3}^{2}\right)^{n} J_{n}^{(p)}\left(x_{3}\right) J_{n}^{(k)}\left(x_{3}\right) d x_{3}=\delta_{p k} h_{k}
$$

with some real coefficients $h_{k}$ (depending on $n$ ). The hierarchical family of extraction polynomials, denoted by $B_{n}^{(k)}\left(x_{3}\right)$, has to be chosen so to satisfy the conditions of Theorem 1 : $B_{n}^{(k)}( \pm 1)=\partial_{3} B_{n}^{(k)}( \pm 1)=\cdots=\partial_{3}^{m-1} B_{n}^{(k)}( \pm 1)=0$. To fulfil this, we choose the specific extraction polynomials denoted in the sequel by $B J$ as:

$$
B J_{n}^{(k)}\left(x_{3}\right)=\left(1-x_{3}^{2}\right)^{n} \frac{J_{n}^{(k)}\left(x_{3}\right)}{h_{k}},
$$

so that, according to (32), we retrieve the coefficients $\tilde{a}_{k}$ in (31) as a simple scalar product:

$$
\int_{-1}^{1} A_{i}\left(x_{3}\right) B J_{n}^{(k)}\left(x_{3}\right) d x_{3}=\tilde{a}_{k} \quad k=0,1, \ldots, N
$$

Thus, by virtue of Theorem 1 , the $J[R]$ integral evaluated for the quasi-dual functions $K_{m}^{\left(\alpha_{i}\right)}[B J]$ with the extraction polynomials $B=B J_{n}^{(k)}, k=0,1, \ldots, N$ provides approximations of the coefficients $\tilde{a}_{k}$. Notice that the notation $B J_{n}^{(k)}$ indicates that the extraction polynomials are based on the Jacobi polynomials, the number of homogeneous derivatives at \pm 1 is the subscript $n$ (the order of the Jacobi polynomial), and the polynomial degree is the superscript $k$. Of course, 
in general $A_{i}\left(x_{3}\right)$ is an unknown function and we wish to find a projection of it into spaces of polynomials. It is expected that as we increase the polynomial space, the approximation is better.

The ESIF $A_{i}\left(x_{3}\right)$ has an infinite Fourier expansion in the basis $J_{n}^{(k)}$ with a sequence of coefficients $\tilde{a}_{k}$ :

$$
A_{i}\left(x_{3}\right)=\sum_{k \geq 0} \tilde{a}_{k} J_{n}^{(k)}
$$

converging in the weighted space $L^{2}[w]$ with $w=\left(1-x_{3}^{2}\right)^{n}$. For each fixed $n$, the computation of the $n+1$ coefficients $\tilde{a}_{0}, \ldots, \tilde{a}_{n}$ provides the orthogonal projection of $A^{\left(\alpha_{i}\right)}\left(x_{3}\right)$ into the space of polynomials of degree up to $n$ in the weighted space $L^{2}[w]$. To accomplish this we use the $n+1$ extraction polynomials $B J_{n}^{(0)}\left(x_{3}\right), \ldots, B J_{n}^{(k)}\left(x_{3}\right)$ defined in (33), so that there holds according to (32):

$$
\int_{-1}^{1} A_{i}\left(x_{3}\right) B J_{n}^{(k)}\left(x_{3}\right) d x_{3}=\tilde{a}_{k} \quad k=0,1, \ldots, N
$$

If we want to increase the space in which $A_{i}\left(x_{3}\right)$ is projected, all which is needed is the computation of (36) for $k=N+1$. This way: $A^{\text {new }}\left(x_{3}\right)=A^{\text {previous }}\left(x_{3}\right)+\tilde{a}_{N+1} J_{N+1}\left(x_{3}\right)$.

\subsection{Jacobi Extraction Polynomials of Order 4}

For the sake of simplicity, the first three dual singular functions $K_{0}^{\left(\alpha_{i}\right)}, K_{1}^{\left(\alpha_{i}\right)}$ and $K_{2}^{\left(\alpha_{i}\right)}$ are considered herein. Thus, according to Theorem 1, it is necessary that the Jacobi extraction polynomials satisfy the conditions in (29) at least to $m=2$. In [12] it was noticed that if the minimal condition is satisfied one does indeed recover the expected rate of convergence in respect with $R$, however, poor results are evident at the two ends of the edge (this behavior was noticed also if the edge portion at which EFIFs was entirely within the domain, i.e. $-0.6<x_{3}<0.6$ ). This phenomenon is attributed to the large values of the derivatives of the Jacobi polynomials at the end points as explained in details in Appendix C. Therefore we select the Jacobi extraction polynomials $B J_{4}^{(k)}$, which satisfy $(29)$ up to $m=4$. The Jacobi extraction polynomials $B J_{4}^{(k)}$ are used for the construction of the dual singular functions $K_{0}^{\left(\alpha_{i}\right)}, K_{1}^{\left(\alpha_{i}\right)}$ and $K_{2}^{\left(\alpha_{i}\right)}$. There holds [2, pp. 773-774]:

$$
J_{4}^{(k)}\left(x_{3}\right)=\frac{(k+4) !}{(k+8) !} \sum_{l=0}^{k} \frac{(k+l+8) !}{2^{l} l !(k-l) !(4+l) !}\left(x_{3}-1\right)^{l}
$$

and the constant $h_{k}$ in (32) is equal to

$$
h_{k}=\frac{2^{9}(k+4) !(k+4) !}{(2 k+9)(k+8) !}
$$

Inserting (38) and (37) in (33), we finally obtain:

$$
B J_{4}^{(k)}\left(x_{3}\right)=\frac{(2 k+9)\left(1-x_{3}^{2}\right)^{4}}{2^{9}(k+4) !} \sum_{l=0}^{k} \frac{(k+l+8) !}{2^{l} l !(k-l) !(4+l) !}\left(x_{3}-1\right)^{l} .
$$




\subsection{Numerical Computation of $J[R]$ Integral}

The exact solution $\tilde{\boldsymbol{u}}$ is in general unknown, so we use instead a finite element approximation $\tilde{\boldsymbol{u}}_{\mathrm{FE}}$ and the integral (27) is computed numerically using a Gaussian quadrature of order $n_{G}$ :

$$
J[R]\left(\tilde{\boldsymbol{u}}, \mathbf{K}_{m}^{\left(\alpha_{i}\right)}[B J]\right)=\sum_{k=1}^{n_{G}} \sum_{\ell=1}^{n_{G}} \frac{\omega}{2} w_{k} w_{\ell}\left([T] \tilde{\boldsymbol{u}}_{\mathrm{FE}} \cdot \mathbf{K}_{m}^{\left(\alpha_{i}\right)}\left[B J_{n}^{(k)}\right]-\tilde{\boldsymbol{u}}_{\mathrm{FE}} \cdot[T] \mathbf{K}_{m}^{\left(\alpha_{i}\right)}\left[B J_{n}^{(k)}\right]\right)_{\xi_{k}, \eta_{\ell}}
$$

where $w_{k}$ are the weights and $\xi_{k}$ and $\eta_{\ell}$ are the abscissas of the Gaussian quadrature. The Neumann trace operator, $[T]$, operates on both $\tilde{\boldsymbol{u}}$ and $\mathbf{K}_{m}^{\left(\alpha_{i}\right)}\left[B J_{n}^{(k)}\right]$. For $T \tilde{\boldsymbol{u}}$ we use the numerical approximations $T \tilde{\boldsymbol{u}}_{\mathrm{FE}}$ computed by finite elements (notice that such extractions are easily computed by the $p$-version of the FEM at any point within an element) whereas $[T] \mathbf{K}_{m}^{\left(\alpha_{i}\right)}\left[B J_{n}^{(k)}\right]$ is computed analytically. These values are evaluated at the specific Gaussian points at which the integral is computed numerically.

\section{Numerical Example - A Cracked Domain $(\omega=2 \pi)$ with Traction Free Boundary Conditions}

We can generate an exact solution to a crack in a three-dimensional isotropic domain with traction free boundary conditions by computing analytically the primal and shadow eigen functions $\boldsymbol{\Phi}_{0}$, $\boldsymbol{\Phi}_{1}, \boldsymbol{\Phi}_{2}$ and the dual and shadow eigen functions $\boldsymbol{\Psi}_{0}, \boldsymbol{\Psi}_{1}, \boldsymbol{\Psi}_{2}$. Their formulas are presented in Appendix A. We refer to the first three eigen-values only where in a case of cracked domain the first three eigen values are identical: $\alpha_{1}=\alpha_{2}=\alpha_{3}=\frac{1}{2}$ and are the only eigen-values which are smaller then 1 . Next we chose the ESIF $A_{i}\left(x_{3}\right)$ to be, for example, a polynomial of order 3 at most, i.e. $A_{i}\left(x_{3}\right)=a_{0}^{(i)}+a_{1}^{(i)} x_{3}+a_{2}^{(i)} x_{3}^{2}$. We obtain therefore an exact solution (16) with a finite number of terms in the sum, because the $3^{\text {rd }}$ and higher derivatives are zero. The exact solution is:

$$
\tilde{\boldsymbol{u}}^{\left(\alpha_{i}\right)}=\sum_{j \geq 0}^{2} \partial_{3}^{j} A_{i}\left(x_{3}\right) r^{\alpha_{i}+j} \boldsymbol{\varphi}_{j}^{\left(\alpha_{i}\right)}(\theta)
$$

Let us consider the following ESIFs (polynomials of order):

$$
A_{1}\left(x_{3}\right)=3+4 x_{3}+5 x_{3}^{2} \quad, \quad A_{2}\left(x_{3}\right)=2+3 x_{3}+4 x_{3}^{2} \quad, \quad A_{3}\left(x_{3}\right)=5+4 x_{3}+2 x_{3}^{2}
$$

The domain has been discretized by using a $p$-FEM mesh, with geometrical progression towards the singular edge with a factor of 0.15 , having 4 layers of elements. In the $x_{3}$ direction, a uniform discretization using 5 elements has been adopted. In Figure 2 we present the mesh used for the cracked domain.

By specifying on the entire boundary $\partial \Omega$ Dirichlet boundary conditions according to the exact solution $\tilde{\boldsymbol{u}}$ (41) of Navier-Lamé operator (2),(3),(4). This way, the exact solution at any point $\boldsymbol{x} \equiv\left(r, \theta, x_{3}\right)$ is therefore (41). In all numerical examples the Young modulus is taken to be 1 and the Poisson ratio 0.3 , so the Lamé constants are $\lambda=0.5769$ and $\mu=0.3846$. 


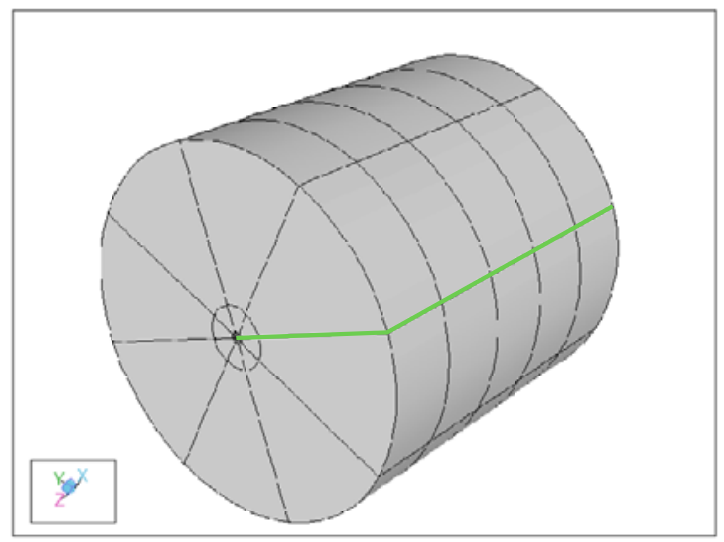

Figure 2. The p-FEM model of the cracked domain.

\subsection{Numerical Tests of J[R] Integral, using $K_{m}^{\left(\alpha_{i}\right)}$}

Computing $J[R]$ for different values of $R$ using the quasidual function $K_{m}^{\left(\alpha_{i}\right)}$ and $B J_{4}^{(k)}\left(x_{3}\right)$ should provide $J[R]=\tilde{a}_{k}^{\left(\alpha_{i}\right)}$ with an error of $\mathcal{O}\left(R^{\alpha_{1}-\alpha_{i}+m+1}\right)$. For a traction free cracked domain $\alpha_{1}=\alpha_{2}=\alpha_{3}=\frac{1}{2}$, and therefore we expect the convergence rate to be at least $\mathcal{O}(R)$ for $K_{0}^{\left(\alpha_{i}\right)}$, $\mathcal{O}\left(R^{2}\right)$ for $K_{1}^{\left(\alpha_{i}\right)}$ and $\mathcal{O}\left(R^{3}\right)$ for $K_{2}^{\left(\alpha_{i}\right)}$ for all first three eigen-pairs.

The numerical tests use the boundary condition (41) with the exact ESIF's as specified in (42), and we compute $J[R]$ at different values of $R$ for $K_{0}^{\left(\alpha_{i}\right)}, K_{1}^{\left(\alpha_{i}\right)}$ and $K_{2}^{\left(\alpha_{i}\right)}$. The Gauss quadrature is of order 10 in both $\theta$ and $x_{3}$ directions and the finite element solution at $p=7$ is used. Taking 32 integration points and $p=8$ does not improve the results considerably.

We plot in figure $3 \log \left(\left(J_{\mathrm{ex}}-J[R]\right) / J_{\mathrm{ex}}\right)$ vis. $\log (R)$, showing the numerical convergence rate. As the finite element solution has a numerical error of about $0.1-1 \%$ in energy norm, the values of $J[R]$ cannot be computed with a better accuracy, therefore, a relative error of $10^{-3}$ is the lower limit expected. We use $B J_{4}^{(0)}\left(x_{3}\right), B J_{4}^{(1)}\left(x_{3}\right)$ and $B J_{4}^{(2)}\left(x_{3}\right)$ for $K_{0}^{\left(\alpha_{i}\right)}, K_{1}^{\left(\alpha_{i}\right)}, K_{2}^{\left(\alpha_{i}\right)}$, so $J[R]$ represents the nine coefficients $\tilde{a}_{i}^{\left(\alpha_{j}\right)}, i=0,1,2, j=1,2,3$.

Because the exact ESIFs are at most polynomials of order 2, the $J[R]$ value for $B J_{4}^{(3)}\left(x_{3}\right)$, $B J_{4}^{(4)}\left(x_{3}\right), B J_{4}^{(5)}\left(x_{3}\right)$ (computed with $K_{2}^{\left(\alpha_{i}\right)}$ ) are expected to be zero and shown in Table I.

We can see in Figure 3 that the convergence rate of $J[R]$ is of order $R^{m+1}$ as we expected.

\subsection{Numerical Computation of ESIFs}

When $J[R]$ is computed with the quasi-dual function $K_{m}^{\left(\alpha_{i}\right)}$ and $B J_{4}^{(k)}\left(x_{3}\right)$ we obtain according to (44) the coefficient $\tilde{a}_{j}^{\left(\alpha_{i}\right)}$ :

$$
J[0]=\int_{-1}^{1} A_{i}\left(x_{3}\right) B J_{4}^{(j)}\left(x_{3}\right) d x_{3}=\tilde{a}_{j}^{\left(\alpha_{i}\right)} \quad j=0,1, \cdots, n
$$



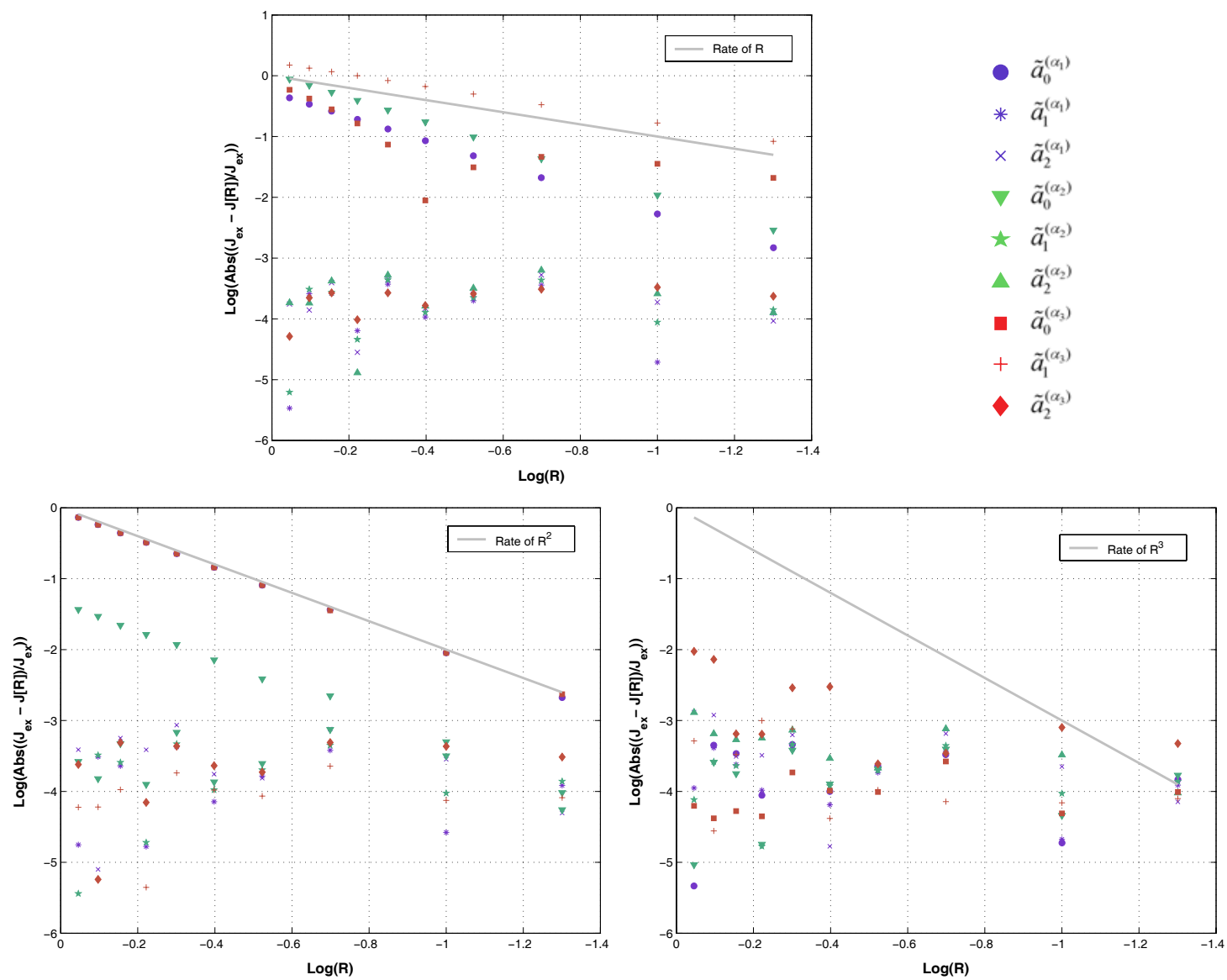

Figure 3. Convergence rates of $J[R]$ (the coefficients of the polynomial describing the ESIFs) using $B J_{4}^{(k)}$, $k=0,1,2$ and $K_{0}^{\left(\alpha_{i}\right)}, K_{1}^{\left(\alpha_{i}\right)}, K_{2}^{\left(\alpha_{i}\right)}$ for the traction free cracked domain.

The ESIF is then easily represented by a linear combination of the Jacobi polynomials as:

$$
A_{i}\left(x_{3}\right)=\tilde{a}_{0} J_{4}^{(0)}\left(x_{3}\right)+\tilde{a}_{1} J_{4}^{(1)}\left(x_{3}\right)+\tilde{a}_{2} J_{4}^{(2)}\left(x_{3}\right)+\cdots
$$

The advantage of the hierarchical family of polynomials is that one can adaptively increase the polynomial order of the ESIF. For example, if one is interested in projecting $A_{i}\left(x_{3}\right)$ into the space of polynomials of degree up to $n$, the $n+1$ coefficients $\tilde{a}_{0}, \cdots, \tilde{a}_{n}$ are being computed using the $n+1$ extraction polynomials $B J_{4}^{(0)}\left(x_{3}\right), \cdots, B J_{4}^{(n)}\left(x_{3}\right)$ defined in (33).

To increase the space in which $A_{i}\left(x_{3}\right)$ is projected, all which is needed is the computation of (40) for $n+1$. This way, the new $A_{i}^{\text {new }}=A_{i}^{\text {previous }}+\tilde{a}_{n+1} J_{4}^{(n+1)}\left(x_{3}\right)$. We illustrate the extracted polynomial representation of the ESIF, $A_{1}\left(x_{3}\right), A_{2}\left(x_{3}\right), A_{3}\left(x_{3}\right)$ of order $2,3,4,5$, and its relative error using the data at $R=0.05$ in Figure 4,5 and 6 respectively and using $K_{2}^{\left(\alpha_{i}\right)}$.

Notice that the relative error of the extracted ESIFs is lower than $0.1 \%$. The results show an accurate and efficient method. 
Table I. Numerical results of $J[R]$ using $B J_{4}^{(k)}, k=3,4,5$ and $K_{2}^{\left(\alpha_{i}\right)}$, for the traction free cracked domain.

\begin{tabular}{|c|c|c|c|c|c|c|}
\hline & \multicolumn{6}{|c|}{$\alpha_{1}=0.5$} \\
\hline$R$ & $J_{e x}$ & $B J_{4}^{(3)}$ & $J_{e x}$ & $B J_{4}^{(4)}$ & $\| J_{e x}$ & $B J_{4}^{(5)}$ \\
\hline 0.9 & 0 & $-1.09 \mathrm{E}-04$ & 0 & $3.18 \mathrm{E}-04$ & 0 & $6.44 \mathrm{E}-05$ \\
\hline 0.8 & 0 & $1.65 \mathrm{E}-04$ & 0 & $7.75 \mathrm{E}-05$ & 0 & $-1.28 \mathrm{E}-04$ \\
\hline 0.7 & 0 & $-4.93 \mathrm{E}-05$ & 0 & $-1.87 \mathrm{E}-04$ & 0 & $3.75 \mathrm{E}-05$ \\
\hline 0.6 & 0 & $-7.77 \mathrm{E}-05$ & 0 & $-1.00 \mathrm{E}-04$ & 0 & $5.18 \mathrm{E}-05$ \\
\hline 0.5 & 0 & $3.35 \mathrm{E}-05$ & 0 & $6.53 \mathrm{E}-05$ & 0 & $-2.14 \mathrm{E}-05$ \\
\hline 0.4 & 0 & $2.81 \mathrm{E}-05$ & 0 & $5.74 \mathrm{E}-05$ & 0 & $-8.31 \mathrm{E}-06$ \\
\hline 0.3 & 0 & $-1.65 \mathrm{E}-05$ & 0 & $-6.23 \mathrm{E}-05$ & 0 & $-1.87 \mathrm{E}-06$ \\
\hline 0.2 & 0 & $2.17 \mathrm{E}-05$ & 0 & $-7.46 \mathrm{E}-06$ & 0 & $-6.25 \mathrm{E}-06$ \\
\hline 0.1 & 0 & $1.98 \mathrm{E}-05$ & 0 & $-3.21 \mathrm{E}-05$ & 0 & $-3.01 \mathrm{E}-05$ \\
\hline 0.05 & 0 & $1.97 \mathrm{E}-05$ & 0 & $1.10 \mathrm{E}-06$ & 0 & $-2.75 \mathrm{E}-05$ \\
\hline
\end{tabular}

\begin{tabular}{|c|c|c|c|c|c|c|c|c|c|c|c|c|}
\hline \multirow[b]{2}{*}{$R$} & \multicolumn{6}{|c|}{$\alpha_{2}=0.5$} & \multicolumn{6}{|c|}{$\alpha_{3}=0.5$} \\
\hline & $J_{e x}$ & $B J_{4}^{(3)}$ & $J_{e x}$ & $B J_{4}^{(4)}$ & $J_{e x}$ & $B J_{4}^{(5)}$ & $J_{e x}$ & $B J_{4}^{(3)}$ & $J_{e x}$ & $B J_{4}^{(4)}$ & $J_{e x}$ & $B J_{4}^{(5)}$ \\
\hline 0.9 & 0 & $1.06 \mathrm{E}-04$ & 0 & $-1.93 \mathrm{E}-04$ & 0 & $-7.91 \mathrm{E}-05$ & 0 & $3.81 \mathrm{E}-04$ & 0 & $1.74 \mathrm{E}-04$ & 0 & $1.42 \mathrm{E}-03$ \\
\hline 0.8 & 0 & $-1.08 \mathrm{E}-04$ & 0 & $3.50 \mathrm{E}-05$ & 0 & $1.05 \mathrm{E}-04$ & 0 & $-6.56 \mathrm{E}-04$ & 0 & $-2.89 \mathrm{E}-04$ & 0 & $-2.62 \mathrm{E}-04$ \\
\hline 0.7 & 0 & $-1.26 \mathrm{E}-05$ & 0 & $9.74 \mathrm{E}-05$ & 0 & $-1.11 \mathrm{E}-05$ & 0 & $4.24 \mathrm{E}-05$ & 0 & $-3.16 \mathrm{E}-04$ & 0 & $-9.26 \mathrm{E}-04$ \\
\hline 0.6 & 0 & $6.24 \mathrm{E}-05$ & 0 & $-3.34 \mathrm{E}-05$ & 0 & $-6.44 \mathrm{E}-05$ & 0 & $8.01 \mathrm{E}-04$ & 0 & $4.44 \mathrm{E}-04$ & 0 & $4.59 \mathrm{E}-04$ \\
\hline 0.5 & 0 & $1.01 \mathrm{E}-05$ & 0 & $-7.81 \mathrm{E}-05$ & 0 & $2.93 \mathrm{E}-06$ & 0 & $1.88 \mathrm{E}-04$ & 0 & $3.00 \mathrm{E}-04$ & 0 & $6.39 \mathrm{E}-04$ \\
\hline 0.4 & 0 & $-2.51 \mathrm{E}-05$ & 0 & $2.76 \mathrm{E}-06$ & 0 & $3.26 \mathrm{E}-05$ & 0 & $-4.26 \mathrm{E}-04$ & 0 & $-1.57 \mathrm{E}-04$ & 0 & $-1.09 \mathrm{E}-04$ \\
\hline 0.3 & 0 & $1.30 \mathrm{E}-05$ & 0 & $2.72 \mathrm{E}-05$ & 0 & $-2.17 \mathrm{E}-05$ & 0 & $-4.16 \mathrm{E}-05$ & 0 & $-2.33 \mathrm{E}-05$ & 0 & $-1.35 \mathrm{E}-04$ \\
\hline 0.2 & 0 & $1.17 \mathrm{E}-05$ & 0 & $-4.06 \mathrm{E}-05$ & 0 & $-2.02 \mathrm{E}-06$ & 0 & $9.19 \mathrm{E}-05$ & 0 & $-1.13 \mathrm{E}-05$ & 0 & $8.38 \mathrm{E}-05$ \\
\hline 0.1 & 0 & $1.98 \mathrm{E}-05$ & 0 & $-3.01 \mathrm{E}-05$ & 0 & $-2.90 \mathrm{E}-05$ & 0 & $6.03 \mathrm{E}-05$ & 0 & $-4.20 \mathrm{E}-06$ & 0 & $-1.65 \mathrm{E}-05$ \\
\hline 0.05 & 0 & $1.82 \mathrm{E}-05$ & 0 & $7.32 \mathrm{E}-06$ & 0 & $-2.28 \mathrm{E}-05$ & 0 & $3.29 \mathrm{E}-05$ & 0 & $-4.39 \mathrm{E}-07$ & 0 & $-1.26 \mathrm{E}-05$ \\
\hline
\end{tabular}
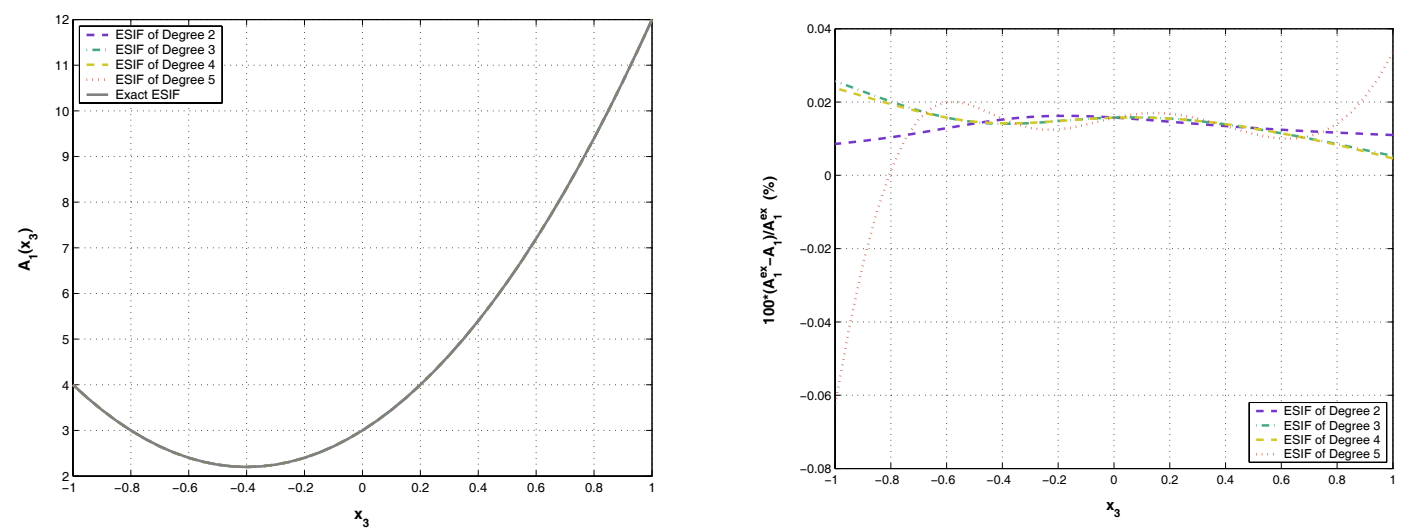

Figure 4. $A_{1}\left(x_{3}\right)$ (left) and its relative error (right) at $R=0.05$. Computations done with $B J_{4}^{(k)}, k=2,3,4,5$, where $A_{1}^{e x}\left(x_{3}\right)=3+4 x_{3}+5 x_{3}^{2}, \omega=2 \pi, \lambda=0.5769$ and $\mu=0.3846$. 

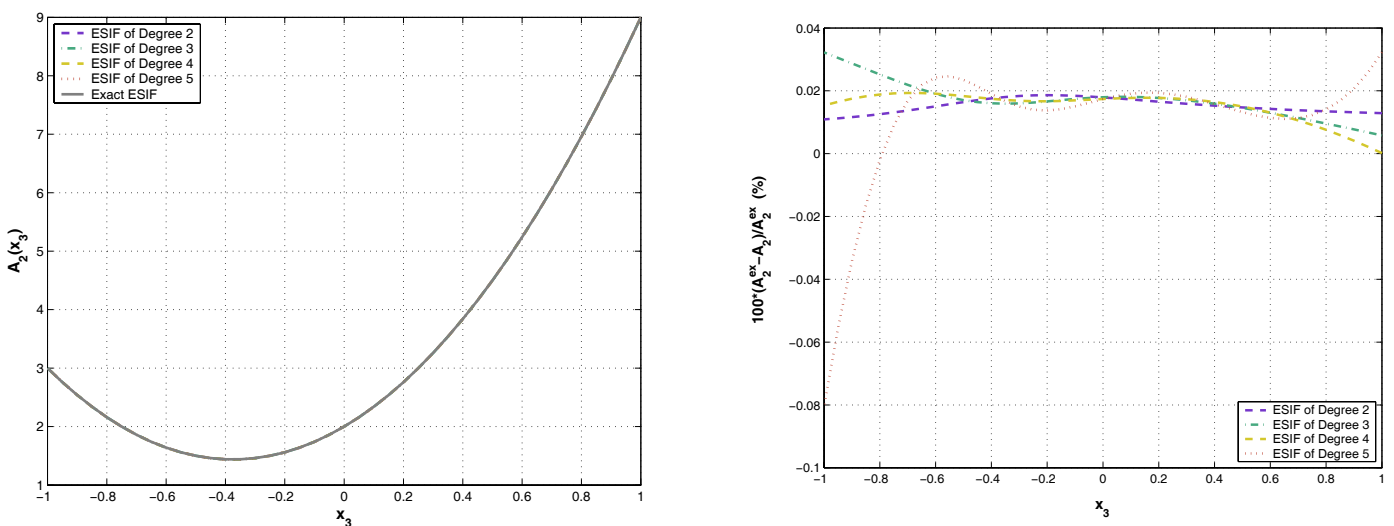

Figure 5. $A_{2}\left(x_{3}\right)$ (left) and its relative error (right) at $R=0.05$. Computations done with $B J_{4}^{(k)}, k=2,3,4,5$, where $A_{2}^{e x}\left(x_{3}\right)=2+3 x_{3}+4 x_{3}^{2}, \omega=2 \pi, \lambda=0.5769$ and $\mu=0.3846$.
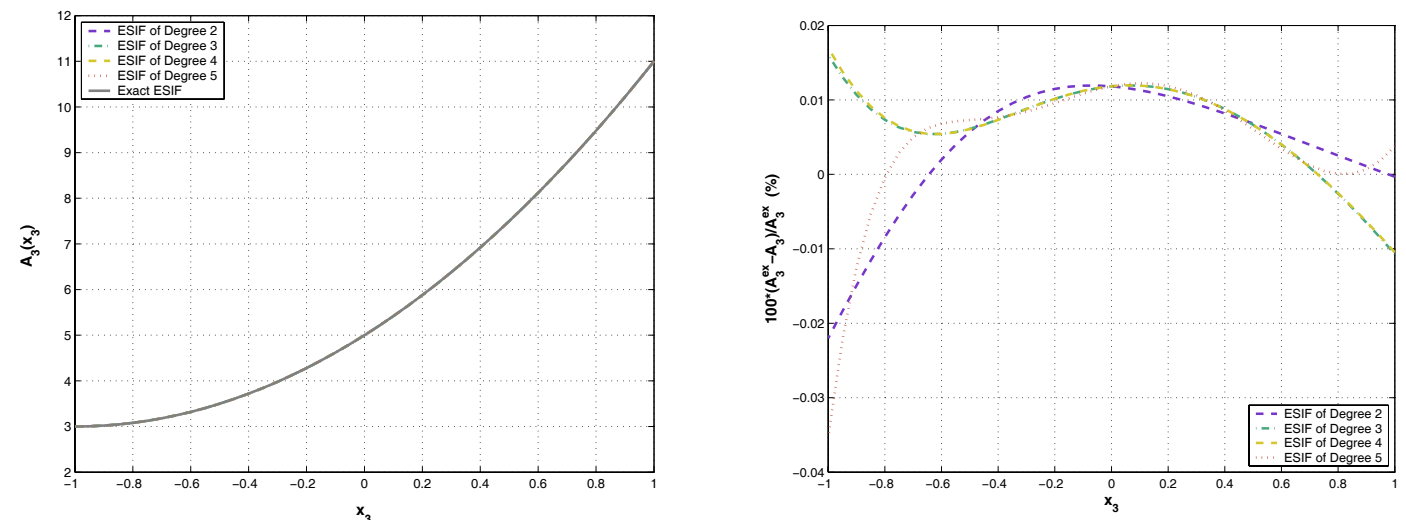

Figure 6. $A_{3}\left(x_{3}\right)$ (left) and its relative error (right) at $R=0.05$. Computations done with $B J_{4}^{(k)}, k=2,3,4,5$, where $A_{3}^{\text {ex }}\left(x_{3}\right)=5+4 x_{3}+2 x_{3}^{2}, \omega=2 \pi, \lambda=0.5769$ and $\mu=0.3846$. 


\section{Numerical Example - Clamped V-Notched Domain $\left(\omega=\frac{3 \pi}{2}\right)$}

Similar to the previous section, we generate an exact solution to a v-notched domain $\left(\omega=\frac{3 \pi}{2}\right)$ with clamped boundary conditions on the surfaces $\Gamma_{1}$ and $\Gamma_{2}$ by computing analytically the primal and shadow eigen functions $\boldsymbol{\Phi}_{0}, \boldsymbol{\Phi}_{1}, \boldsymbol{\Phi}_{2}$ and the dual and shadow eigen functions $\boldsymbol{\Psi}_{0}$, $\boldsymbol{\Psi}_{1}, \boldsymbol{\Psi}_{2}$. Their formulae are presented in Appendix B.

We select the ESIF to be polynomials of order 2 as presented in (42), such that the the exact solution (16) contain only three terms in the sum, (41).

The domain $\omega=\frac{3 \pi}{2}$ has been discretized by using a $p$-FEM mesh, with geometrical progression towards the singular edge with a factor of 0.15 , having 4 layers of elements. In the $x_{3}$ direction, a uniform discretization using 5 elements has been adopted, as presented in Figure 7. We specify over the entire boundary $\partial \Omega$ displacements boundary conditions according to the

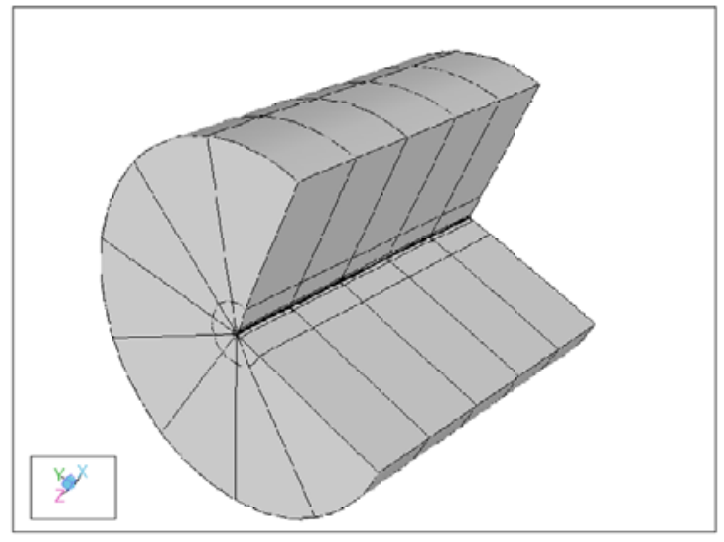

Figure 7. The $p$-FEM model of the $\omega=\frac{3 \pi}{2} \quad V$-notched domain.

exact solution $\tilde{\boldsymbol{u}}(41)$. The FE solution at any point $\boldsymbol{x} \equiv\left(r, \theta, x_{3}\right)$ is therefore the exact solution (41).

\subsection{Numerical Tests of J[R] Integral, using $K_{m}^{\left(\alpha_{i}\right)}$}

We again compute $J[R]$ for different values of $R$ using the quasidual function $K_{m}^{\left(\alpha_{i}\right)}$ and $B J_{4}^{(k)}\left(x_{3}\right)$ similarly to the case of the crack. In this case the first three eigen-values are distinct, $\alpha_{1}=0.595156, \alpha_{2}=0.759042, \alpha_{3}=0.666667$ and therefore we expect the convergence rate of $J[R]$ for:

- $\quad \alpha_{1}$ to be at least $\mathcal{O}(R), \mathcal{O}\left(R^{2}\right)$ and $\mathcal{O}\left(R^{3}\right)$ when using $K_{0}^{\left(\alpha_{i}\right)}, K_{1}^{\left(\alpha_{i}\right)}$, and $K_{2}^{\left(\alpha_{i}\right)}$ respectively

- $\quad \alpha_{2}$ to be at least $\mathcal{O}\left(R^{0.83611}\right), \mathcal{O}\left(R^{1.83611}\right)$ and $\mathcal{O}\left(R^{2.83611}\right)$ when using $K_{0}^{\left(\alpha_{i}\right)}, K_{1}^{\left(\alpha_{i}\right)}$, and $K_{2}^{\left(\alpha_{i}\right)}$ respectively 
- $\quad \alpha_{3}$ to be at least $\mathcal{O}\left(R^{0.92848}\right), \mathcal{O}\left(R^{1.92848}\right)$ and $\mathcal{O}\left(R^{2.92848}\right)$ when using $K_{0}^{\left(\alpha_{i}\right)}, K_{1}^{\left(\alpha_{i}\right)}$, and $K_{2}^{\left(\alpha_{i}\right)}$ respectively

We compute $J[R]$ at different values of $R$ for $K_{0}^{\left(\alpha_{i}\right)}, K_{1}^{\left(\alpha_{i}\right)}$ and $K_{2}^{\left(\alpha_{i}\right)}$. The Gauss quadrature is of order 10 in both $\theta$ and $x_{3}$ directions and the finite element solution at $p=7$ is used.

We plot in figure $8 \log \left(\left(J_{\mathrm{ex}}-J[R]\right) / J_{\mathrm{ex}}\right)$ vis. $\log (R)$, showing the numerical convergence rate. As the finite element solution has a numerical error of about $0.1-1 \%$ in energy norm, the values of $J[R]$ cannot be computed with a better accuracy, therefore, a relative error of $10^{-3}$ is the lower limit expected. We use $B J_{4}^{(0)}\left(x_{3}\right), B J_{4}^{(1)}\left(x_{3}\right)$ and $B J_{4}^{(2)}\left(x_{3}\right)$ for $K_{0}^{\left(\alpha_{i}\right)}, K_{1}^{\left(\alpha_{i}\right)}, K_{2}^{\left(\alpha_{i}\right)}$, so $J[R]$ represents the nine coefficients $\tilde{a}_{i}^{\left(\alpha_{j}\right)}, i=0,1,2, j=1,2,3$.

Because the exact ESIFs are at most polynomials of order 2, the $J[R]$ value for $B J_{4}^{(3)}\left(x_{3}\right)$, $B J_{4}^{(4)}\left(x_{3}\right), B J_{4}^{(5)}\left(x_{3}\right)$ (computed with $K_{2}^{\left(\alpha_{i}\right)}$ ) are close to zero and have typical values similar to the ones in Table I so are not repeated.
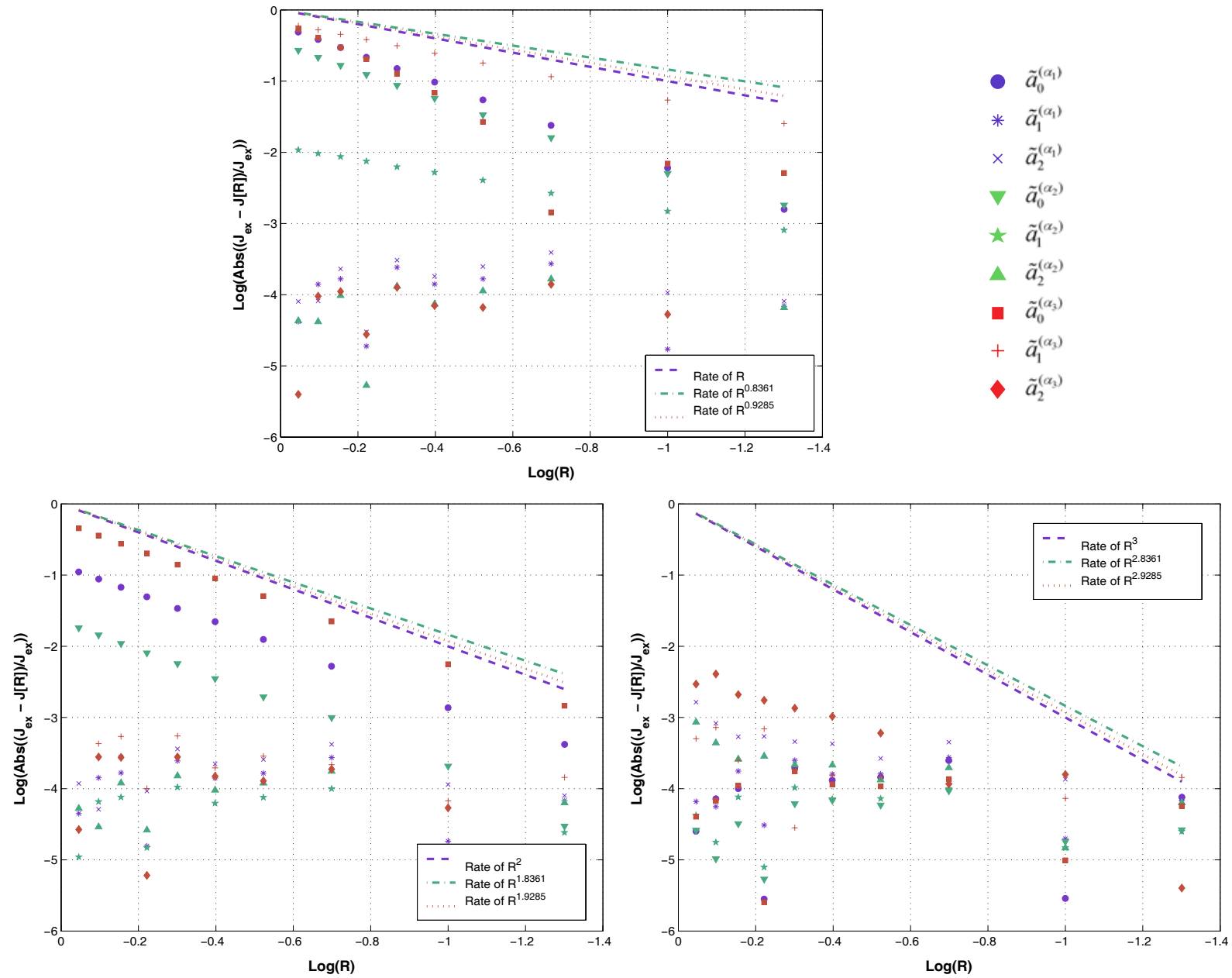

Figure 8. Convergence rates of $J[R]$ (the coefficients of the polynomial describing the ESIFs) using BJ $J_{4}^{(k)}$, $k=0,1,2$ and $K_{0}^{\left(\alpha_{i}\right)}, K_{1}^{\left(\alpha_{i}\right)}, K_{2}^{\left(\alpha_{i}\right)}$ for the clamped $V$-notched domain. 
One may notice in Figure 8 that the convergence rate of $J[R]$ is at least of order $R^{m+1}$ as we expected.

\subsection{Numerical Computation of ESIFs}

After computing the $J[R]$ integrals, the computation of the polynomial representation of the ESIF is simple, using a linear combination of the Jacobi polynomials (44). We illustrate the extracted polynomial representation of the ESIF, $A_{1}\left(x_{3}\right), A_{2}\left(x_{3}\right), A_{3}\left(x_{3}\right)$ and their relative errors using the data at $R=0.05$ in Figure 9,10 and 11 respectively, using $K_{2}^{\left(\alpha_{i}\right)}$.
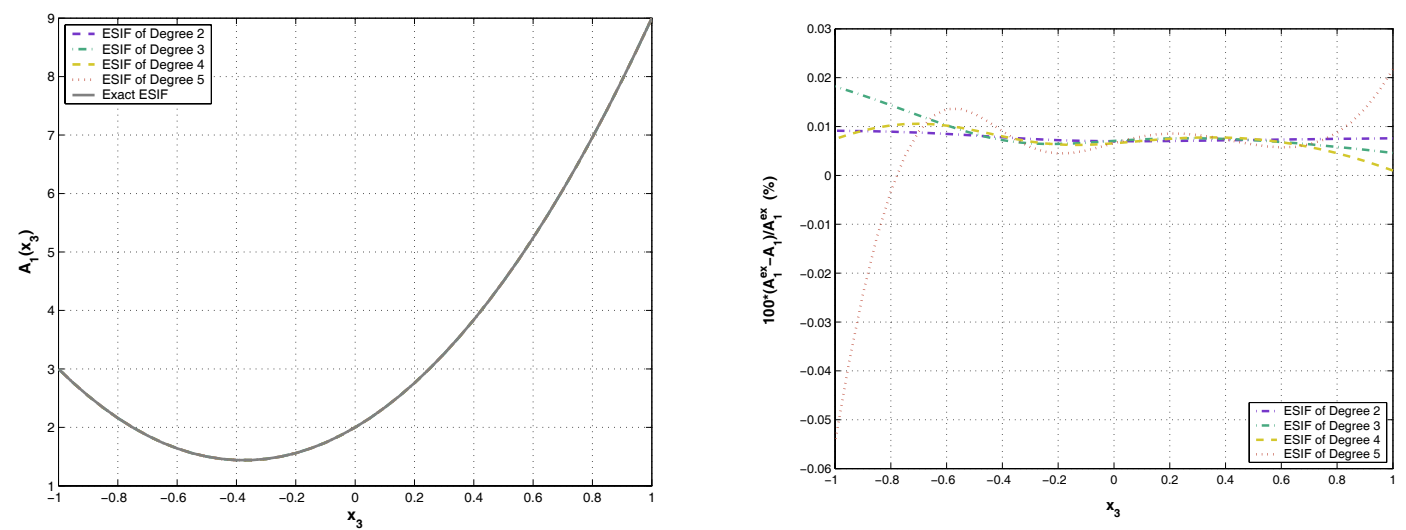

Figure 9. $A_{1}\left(x_{3}\right)$ (left) and its relative error (right) at $R=0.05$ using $B J_{4}^{(k)}, k=2,3,4,5$, where $A_{1}^{e x}\left(x_{3}\right)=3+4 x_{3}+5 x_{3}^{2}, \omega=\frac{3 \pi}{2}, \lambda=0.5769$ and $\mu=0.3846$.
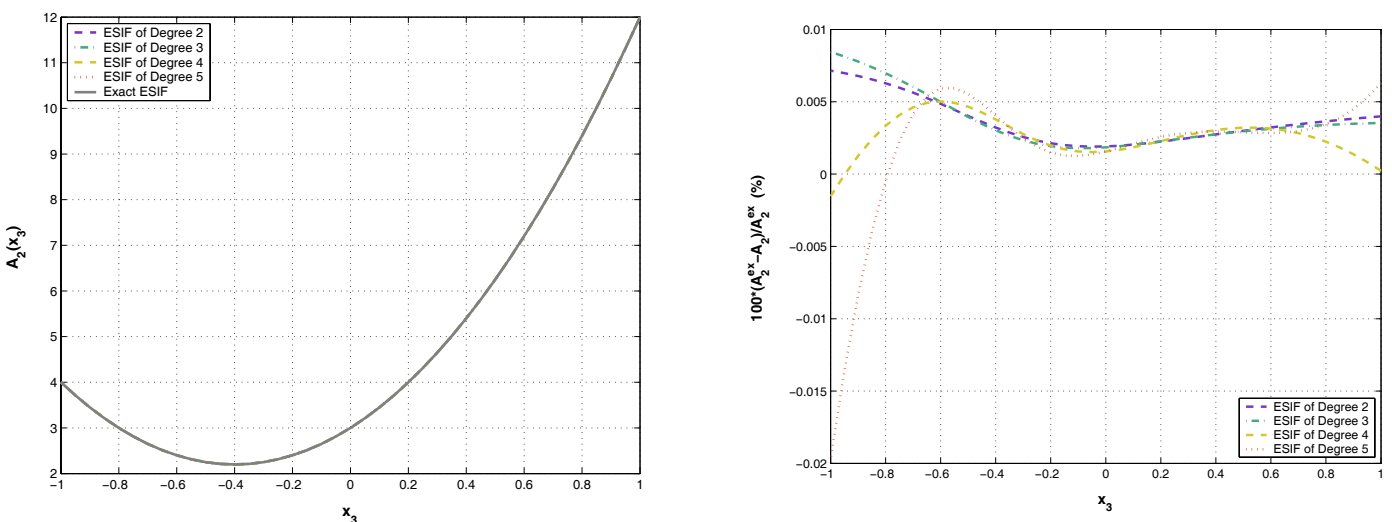

Figure 10. $A_{2}\left(x_{3}\right)$ (left) and its relative error (right) at $R=0.05$ using $B J_{4}^{(k)}, k=2,3,4,5$, where $A_{1}^{e x}\left(x_{3}\right)=2+3 x_{3}+4 x_{3}^{2}, \omega=\frac{3 \pi}{2}, \lambda=0.5769$ and $\mu=0.3846$.

The relative error of the extracted ESIF is not higher than $0.1 \%$. 

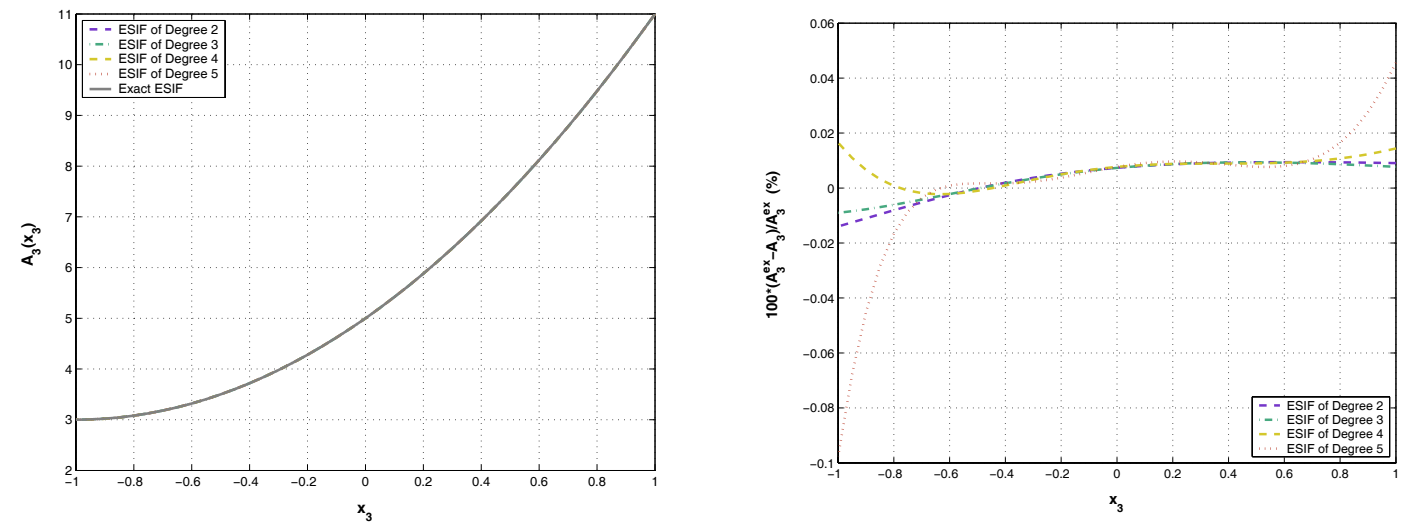

Figure 11. $A_{3}\left(x_{3}\right)$ (left) and its relative error (right) at $R=0.05$ using $B J_{4}^{(k)}, \quad k=2,3,4,5$, where $A_{3}^{e x}\left(x_{3}\right)=5+4 x_{3}+2 x_{3}^{2}, \omega=\frac{3 \pi}{2}, \lambda=0.5769$ and $\mu=0.3846$. 


\section{Compact Tension Specimen - An Example Problem of Engineering Importance}

In this section we compare the ESIFs computed by the quasi-dual function method with a pointwise extraction method of stress intensity factors (SIFs - $K_{I}$ and $K_{I I}$ ) available in [1]. In the classical fracture-mechanics literature the plane-strain SIFs are reported, which multiply a specific "mode I" or "mode II" eigen-functions. To compare between the ESIFs and the SIFs, we first present the relationship between the functions $A_{1}, A_{2}$ and the SIFs $K_{I}$ and $K_{I I}$. We then describe the compact tension specimen (CTS) used for determination of fracture toughness. For the CTS we extract the ESIF using the quasi-dual function method and pointwise values of SIFs and compare between them.

\subsection{The Relation Between the SIFs $K_{I}, K_{I I}$ AND the ESIF}

Under the assumption of plane-strain and mode I loading, the classical solution $\boldsymbol{u}$ in the vicinity of a crack edge is (see e.g. [9]):

$$
\left\{\begin{array}{l}
u_{1} \\
u_{2}
\end{array}\right\}=\frac{K_{I}\left(x_{3}\right)}{2 \mu} \sqrt{\frac{r}{2 \pi}}\left\{\begin{array}{l}
\cos ((\theta+\pi) / 2)\left[\kappa-1+2 \sin ^{2}((\theta+\pi) / 2)\right] \\
\sin ((\theta+\pi) / 2)\left[\kappa+1-2 \cos ^{2}((\theta+\pi) / 2)\right]
\end{array}\right\}
$$

where $\kappa=3-4 \nu$. In the case of plane-strain assumption and mode II loading the classical solution $\boldsymbol{u}$ in the vicinity of a crack edge is:

$$
\left\{\begin{array}{l}
u_{1} \\
u_{2}
\end{array}\right\}=\frac{K_{I I}\left(x_{3}\right)}{2 \mu} \sqrt{\frac{r}{2 \pi}}\left\{\begin{array}{l}
\sin ((\theta+\pi) / 2)\left[\kappa+1+2 \cos ^{2}((\theta+\pi) / 2)\right] \\
\cos ((\theta+\pi) / 2)\left[\kappa-1-2 \sin ^{2}((\theta+\pi) / 2)\right]
\end{array}\right\}
$$

Comparing the displacements expressed above with these expressed in terms of the ESIFs (for $\lambda=0.5769$ and $\mu=0.3846$, see Appendix A), the relation between $A_{1}$ and $K_{I}$ and the relation between $A_{2}$ and $K_{I I}$ in the case of plane strain is:

$$
\begin{aligned}
& \frac{K_{I}}{0.7692 \sqrt{2 \pi}} \cos ((\theta+\pi) / 2)\left[0.8+2 \sin ^{2}((\theta+\pi) / 2)\right]=A_{1}\left[-2.6 \sin \left(\frac{1}{2} \theta\right)-\sin \left(\frac{3}{2} \theta\right)\right] \\
& \frac{K_{I I}}{0.7692 \sqrt{2 \pi}} \sin ((\theta+\pi) / 2)\left[2.8+2 \cos ^{2}((\theta+\pi) / 2)\right]=A_{2}\left[2.2 \cos \left(\frac{1}{2} \theta\right)-\frac{1}{3} \cos \left(\frac{3}{2} \theta\right)\right]
\end{aligned}
$$

which after algebraic manipulation is shown to be independent of $\theta$ :

$$
A_{1}=0.259312 K_{I} \quad, \quad A_{2}=0.777938 K_{I I}
$$

REMARK 3. The strain component $\varepsilon_{33}$ computed using the displacements in (53), for the case $A_{1}$ is a constant is:

$$
\varepsilon_{33}=\frac{\partial^{2} u_{3}}{\partial x_{3}^{2}}=0
$$

On the other hand if plane-stress condition is assumed $\varepsilon_{33}$ is given by:

$$
\varepsilon_{33}=\frac{\sigma_{11}}{E}-\frac{\nu}{E}\left(\sigma_{11}+\sigma_{22}\right) \quad \Rightarrow \quad \varepsilon_{33}=-\frac{\nu}{E}\left(\sigma_{11}+\sigma_{22}\right)=-0.923076 r^{-\frac{1}{2}} \sin \left(\frac{1}{2} \theta\right)
$$

and therefore in $3-D$ the plane-stress condition can not be represented in the vicinity of a singular edge. 


\subsection{Compact Tension Specimen (CtS) Under a Constant Tension Along $x_{3}$}

Consider the classical compact tension specimen (see Figure 12) under bearing loads at the tearing holes having an equivalent force in the $x_{2}$ direction and constant in $x_{3}$ direction, as presented in Figure 13. All other faces are traction free. The thickness of the specimen is 2 ranging from $-1<x_{3}<1$. The specimen is subjected to a tension load of $100[N]$ such that only Mode $I$ is excited along the crack front. Although the loading is independent of $x_{3}$, because of the vertex singularities at $x_{3}= \pm 1$ we anticipate to see a variation in $A_{1}$ as the vertices are approached. The domain is discretized by using a $p$-FEM mesh, with geometrical progression towards the singular edge with a factor of 0.15 where the smallest layer in the vicinity of the edge is at $r=0.15^{3}$. In $x_{3}$ direction we also used a mesh graded in a geometrical progression close to the vertex singularity at $x_{3}= \pm 1$. Smallest layer in the vicinity of the vertex is $-1<x_{3}<-1+0.15^{2}$, $1<x_{3}<1-0.15^{2}$.

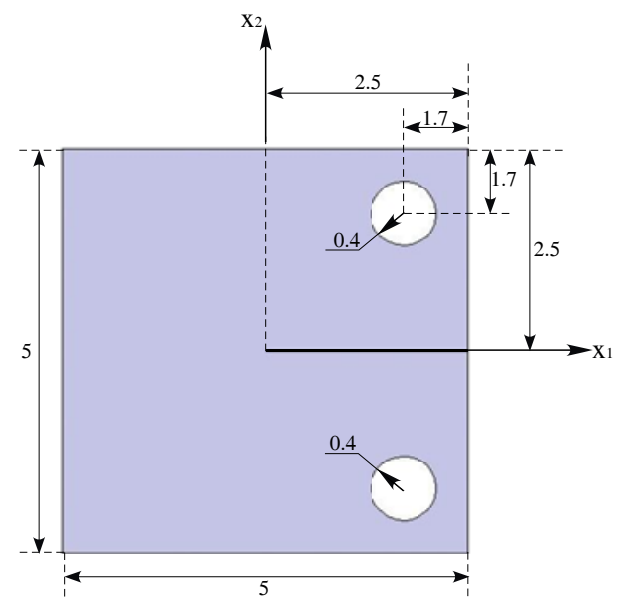

Figure 12. Dimensions of CTS. The thickness of the specimen is 2 ranging from $-1<x_{3}<1$.

We extract the ESIF $A_{1}, A_{2}$ and $A_{3}$ as polynomials of degree 4 and 5 at $R=0.05 . A_{2}$ and $A_{3}$ are of order of $10^{-3}$ (the exact value is zero except maybe at the vertices), which is negligible compared to $A_{1}$, and therefore not plotted herein. The difference in $A_{1}$ as the polynomial degree is increased from 4 to 5 is shown in Figure 14. It may be noticed that the difference between the approximation of $4^{\text {th }}$ and $5^{\text {th }}$ order polynomial is negligible and we use in the sequel polynomial degree 5 for approximating $A_{1}$. Next we compute $A_{1}$ and $K_{I}$ (extracted by the pointwise contour integral method see [1], at several points along the edge) at $R=0.5, R=0.3, R=0.2$, and $R=0.05$ and plot these in Figure 15. We also apply Richardson's extrapolation for $A_{1}$, based on the data at $R=0.5, R=0.3, R=0.2$, shown in Figure 15 .

One may notice the good convergence of the ESIF as $R \rightarrow 0$ compared to the point-wise SIFs.

Next, we wish to demonstrate that the ESIFs can be used away from the singular edge, so a coarse mesh is sufficient. We use the same model with a coarse grid in the vicinity of the edge where the smallest layer in the vicinity of the edge is at $r=0.15$. In $x_{3}$ direction same discretization as in the fine is employed, and perform a FE analysis, using the trunk space up to $p=7$, having 


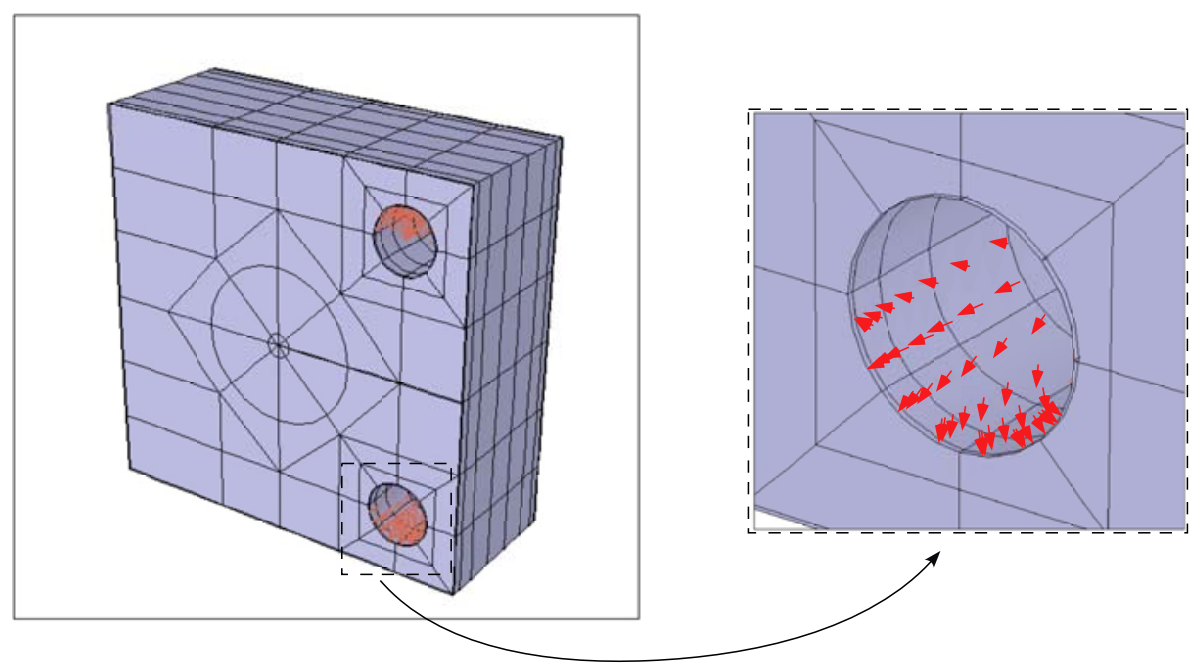

Figure 13. The $p$-FEM model of the CTS with a constant loading in $x_{3}$ direction (the loading at the upper hole is as in the shown lower hole, in the opposite direction).

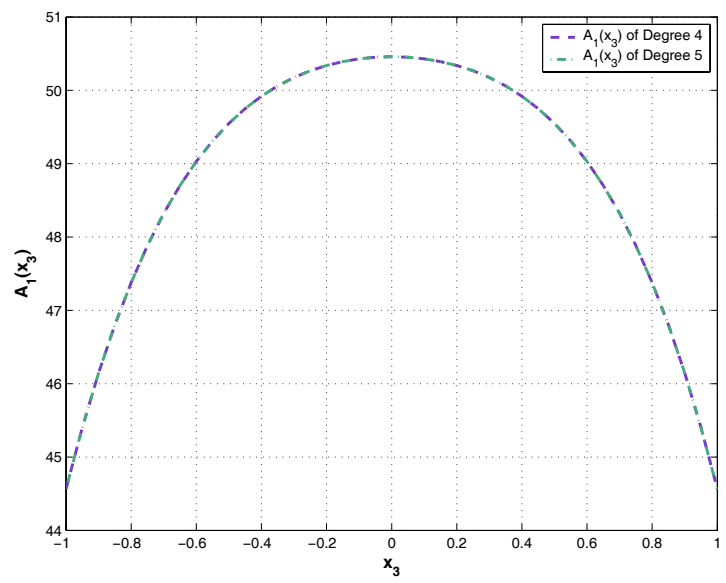

Figure 14. $A_{1}$ extracted at $R=0.05$ using polynomials of degree up to 4 and up to 5 for the CTS.

125,442 DOF. The computed $A_{1}\left(x_{3}\right)$ function and the point-wise values of $K_{I}$ at $R=0.5$, $R=0.3$ and $R=0.2$ are presented in Figure 16. We also apply Richardson's extrapolation for $A_{1}$, based on the data at $R=0.5, R=0.3, R=0.2$, and present it in Figure 16. Although the loading is constant in $x_{3}$, the vertex singularities influence the the ESIF, and as seen usually in practice the crack propagation in the middle of the specimen is usually faster than at the outer surfaces. The results obtained using ESIF extraction method are generated faster than point-wise extraction methods ( $K_{I}$ extraction) and do not require plane stress or plane strain assumptions.

Moreover, we can see that by using Richardson extrapolation using $R=0.5, R=0.3$ and $R=0.2$ we obtain good results, thus we may use a much coarser mesh in the vicinity of the singular edge. It is easy to see that the results of the extracted ESIF using the coarse grid with 125,442 DOF are similar to the results obtained using the refined grid with 150,726 DOF. 


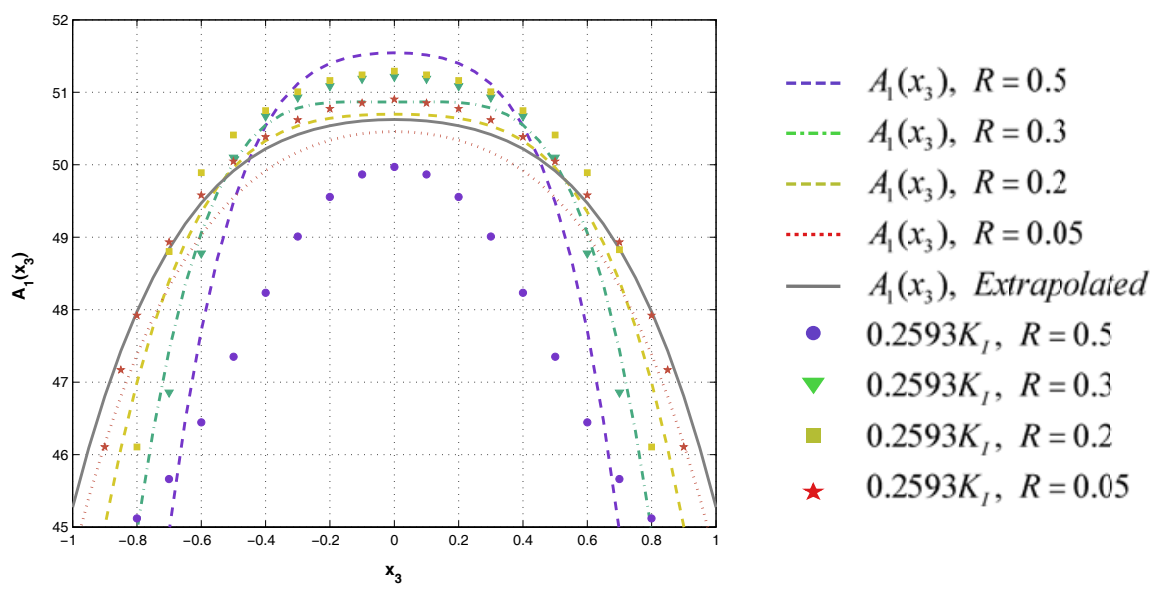

Figure 15. $A_{1}\left(x_{3}\right)$ and $K_{I}$ extracted using different $R$ 's for the compact tension specimen.

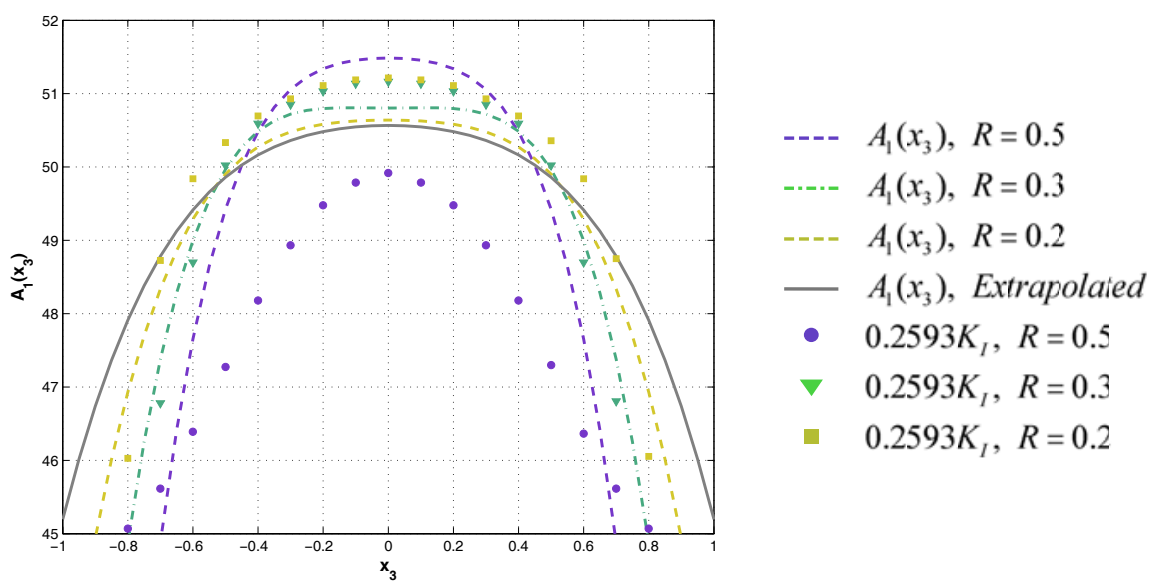

Figure 16. $A_{1}\left(x_{3}\right)$ and $K_{I}$ extracted using different $R$ 's for the compact tension specimen using coarse grid with 125442 DOF.

\section{Summary and Conclusions.}

The quasi-dual function extraction method presented herein is based on the mathematical framework in [5]. The implementation of the method on the EFIF extraction was presented in [12] and the results presented in this paper provide a natural extension to the elasticity system where the method has been slightly improved and realistic engineering problems addressed. Herein we also address cases when both "mode I", "mode II" and "mode III" are excited. The method provides a functional (polynomial) representation of the ESIF along the edge. This accurate and efficient method is implemented as a post-solution operation in conjunction with the $p$-version finite element method.

A hierarchical family of extraction polynomials was constructed, based on Jacobi orthogonal polynomials. The quasi-dual function method, with the use of the "hierarchial family of extraction polynomials" becomes adaptive in the sense that it uses a simple procedure to increase the degree of the extracted ESIF polynomial, thus enable a reliable and efficient determination of ESIFs. 
Analytical solutions have been constructed for cracked and V-notched domains, against which the extracted ESIFs were compared. As shown, the relative errors of the extracted ESIF were less than 1\%, when the degree of the extracted ESIF polynomials is determined by an adaptive procedure.

We also compared the extracted ESIF with point-wise solutions of $K_{I}$ and $K_{I I}$ in the case of a compact tension specimen subject to Mode $I$ loading. It has been shown that the extracted ESIF converge to the solution faster than the stress intensity coefficient $K_{I}$, as $R$ is decreased.

The results presented herein indicate that the method proposed for ESIF extraction is accurate and efficient.

\section{Appendix A}

\section{Primal and Dual Eigen- and Shadow-Functions for a Traction Free Crack.}

The displacements $\tilde{\boldsymbol{u}}(17)$ in the case of a cracked domain $(\omega=2 \pi)$ with traction free boundary conditions on the crack surfaces $\Gamma_{1}$ and $\Gamma_{2}$ is constructed by the primal and shadow functions $\boldsymbol{\Phi}_{j}, j \geq 0 . \boldsymbol{\Phi}_{0}$ and $\boldsymbol{\Psi}_{0}$ are the solutions of the first differential equation of (11). The boundary conditions applied on $\boldsymbol{\Phi}_{0}$ and $\boldsymbol{\Psi}_{0}$ are prescribed in the first equation of (22). There are an infinite number of eigen-values $\alpha_{i}$ for which there is an associated $\boldsymbol{\Phi}_{0}^{\left(\alpha_{i}\right)}$ and $\boldsymbol{\Psi}_{0}^{\left(\alpha_{i}\right)}$ where the positive $\alpha_{i}$ 's are associated with $\boldsymbol{\Phi}_{0}^{\left(\alpha_{i}\right)}$ and the negative $\alpha_{i}$ 's are associated with $\Psi_{0}^{\left(\alpha_{i}\right)}$. We consider the first three eigen-value only equal to $\frac{1}{2}\left(\alpha_{1}=\alpha_{2}=\alpha_{3}=\frac{1}{2}\right)$. The dual eigen function $\Psi_{0}^{\left(\alpha_{i}\right)}$ includes the normalization factor $c_{0}^{\left(\alpha_{i}\right)}$ chosen such that the primal and dual eigen-function, satisfy the orthonormal condition:

$$
\int_{0}^{\omega}\left[[T] \mathbf{\Phi}_{0}^{\left(\alpha_{i}\right)} \cdot \mathbf{\Psi}_{0}^{\left(\alpha_{i}\right)}-\mathbf{\Phi}_{0}^{\left(\alpha_{i}\right)} \cdot[T] \mathbf{\Psi}_{0}^{\left(\alpha_{i}\right)}\right] R d \theta=1
$$

After the primal eigen-functions $\boldsymbol{\Phi}_{0}^{\left(\alpha_{i}\right)}$ and the dual eigen-function $\boldsymbol{\Psi}_{0}^{\left(\alpha_{i}\right)}$ are computed, the first shadow function $\boldsymbol{\Phi}_{1}^{\left(\alpha_{i}\right)}$ and the first dual shadow function $\boldsymbol{\Psi}_{1}^{\left(\alpha_{i}\right)}$ may be computed by the second differential equation in (11), with the second equation of (22) as the boundary conditions. The boundary conditions contain the operators $\left[T_{0}\right]$ and $\left[T_{1}\right]$, as defined in $(23)$

The shadow function $\boldsymbol{\Phi}_{2}^{\left(\alpha_{i}\right)}$ and the dual shadow function $\boldsymbol{\Psi}_{2}^{\left(\alpha_{i}\right)}$ are the solution of the third equation in (11), with the second equation of (22) as the boundary conditions.

The primal solution $\boldsymbol{\Phi}_{0}^{\left(\alpha_{1}\right)}$ in the case of a crack is known as mode $I$ solution. The eigen-value in the case is $\alpha_{1}=1 / 2$ and the primal and shadow functions for $\lambda=0.5769$ and $\mu=0.3846$ are:

$$
\begin{gathered}
\boldsymbol{\Phi}_{0}^{\left(\alpha_{1}\right)}(r, \theta)=r^{\frac{1}{2}}\left(\begin{array}{c}
2.6 \sin \left(\frac{1}{2} \theta\right)+\sin \left(\frac{3}{2} \theta\right) \\
4.6 \cos \left(\frac{1}{2} \theta\right)+\cos \left(\frac{3}{2} \theta\right) \\
0
\end{array}\right) \quad, \quad \boldsymbol{\Phi}_{1}^{\left(\alpha_{1}\right)}(r, \theta)=r^{\frac{3}{2}}\left(\begin{array}{c}
0 \\
0 \\
-2 \sin \left(\frac{1}{2} \theta\right)-3.06667 \sin \left(\frac{3}{2} \theta\right)
\end{array}\right) \\
\mathbf{\Phi}_{2}^{\left(\alpha_{1}\right)}(r, \theta)=r^{\frac{5}{2}}\left(\begin{array}{c}
0.23333 \sin \left(\frac{1}{2} \theta\right)+0.65644 \sin \left(\frac{3}{2} \theta\right) \\
-0.76667 \cos \left(\frac{1}{2} \theta\right)+0.03244 \cos \left(\frac{3}{2} \theta\right) \\
0
\end{array}\right)
\end{gathered}
$$


and the dual shadow functions are:

$$
\begin{gathered}
\Psi_{0}^{\left(-\alpha_{1}\right)}(r, \theta)=0.05542 r^{-\frac{1}{2}}\left(\begin{array}{c}
\sin \left(\frac{1}{2} \theta\right)+1.53333 \sin \left(\frac{3}{2} \theta\right) \\
\cos \left(\frac{1}{2} \theta\right)+0.86667 \cos \left(\frac{3}{2} \theta\right) \\
0
\end{array}\right) \quad, \quad \Psi_{1}^{\left(-\alpha_{1}\right)}(r, \theta)=0.05542 r^{\frac{1}{2}}\left(\begin{array}{c}
0 \\
0 \\
-1.73333 \sin \left(\frac{1}{2} \theta\right)-0.66667 \sin \left(\frac{3}{2} \theta\right)
\end{array}\right) \\
\Psi_{2}^{\left(-\alpha_{1}\right)}(r, \theta)=0.05542 r^{\frac{3}{2}}\left(\begin{array}{c}
0.23778 \sin \left(\frac{1}{2} \theta\right)-0.1 \sin \left(\frac{3}{2} \theta\right) \\
0.495556 \cos \left(\frac{1}{2} \theta\right)-0.43333 \cos \left(\frac{3}{2} \theta\right) \\
0
\end{array}\right)
\end{gathered}
$$

The primal and dual eigen- and shadow-functions associated with $\alpha_{1}=1 / 2$ are presented in figure 17 .
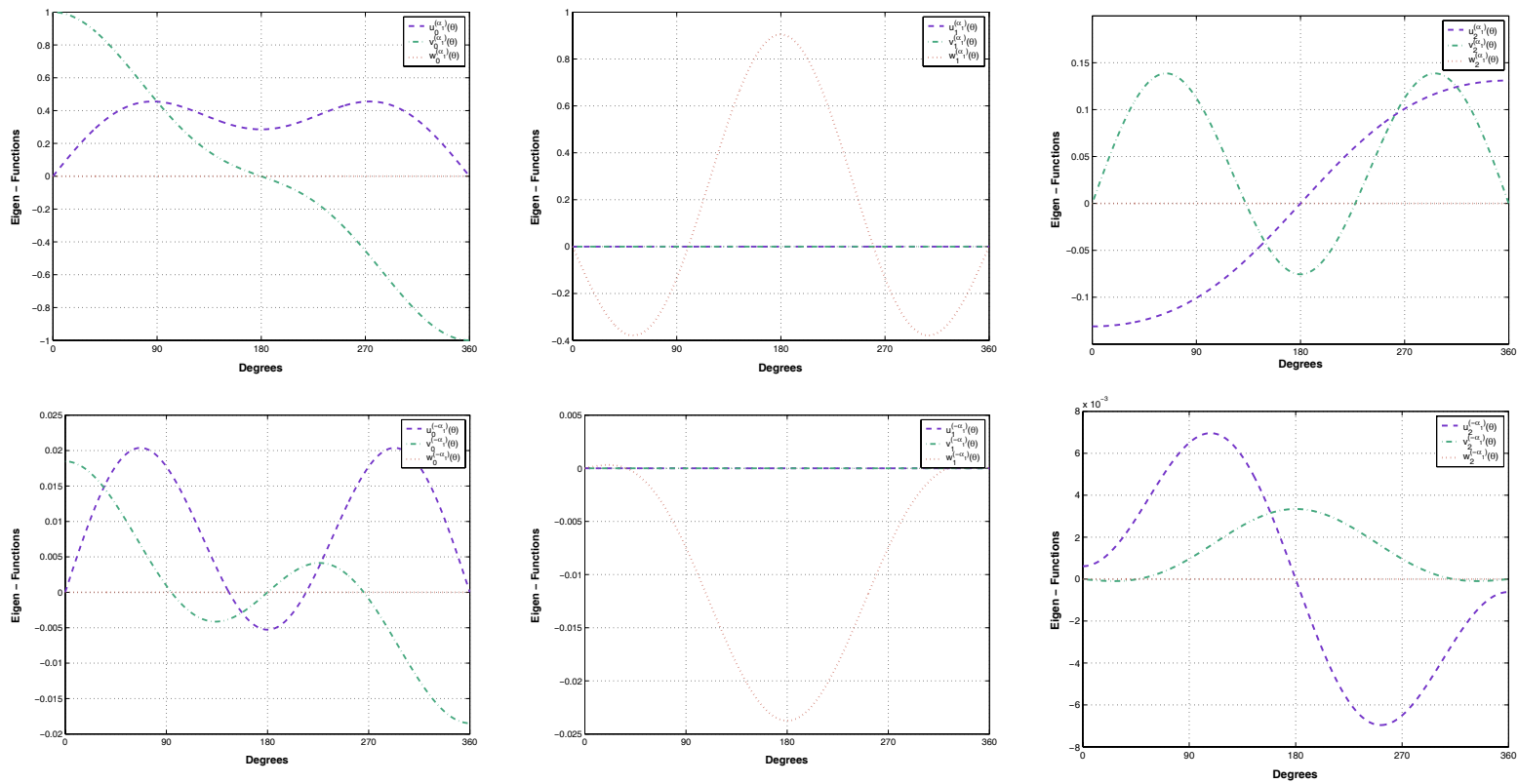

Figure 17. The eigen-functions (Top) and the dual eigen-functions (Bottom) associated with $\alpha_{1}=\frac{1}{2}$ in the case of cracked domain $(\omega=2 \pi), \lambda=0.5769$ and $\mu=0.3846$.

The primal solution $\boldsymbol{\Phi}_{0}^{\left(\alpha_{2}\right)}$ in the case of a crack is known as mode $I I$ solution. The eigen-value in the case is $\alpha_{2}=1 / 2$ and the primal and shadow functions where $\lambda=0.5769$ and $\mu=0.3846$ are:

$$
\begin{gathered}
\Phi_{0}^{\left(\alpha_{2}\right)}(r, \theta)=r^{\frac{1}{2}}\left(\begin{array}{c}
0.86667 \cos \left(\frac{1}{2} \theta\right)+\cos \left(\frac{3}{2} \theta\right) \\
-1.53333 \sin \left(\frac{1}{2} \theta\right)-\sin \left(\frac{3}{2} \theta\right) \\
0
\end{array}\right), \quad \Phi_{1}^{\left(\alpha_{2}\right)}(r, \theta)=r^{\frac{3}{2}}\left(\begin{array}{c}
0 \\
0 \\
-0.66667 \cos \left(\frac{1}{2} \theta\right)
\end{array}\right) \\
\Phi_{2}^{\left(\alpha_{2}\right)}(r, \theta)=r^{\frac{5}{2}}\left(\begin{array}{c}
0.07778 \cos \left(\frac{1}{2} \theta\right)-0.07956 \cos \left(\frac{3}{2} \theta\right) \\
0.25556 \sin \left(\frac{1}{2} \theta\right)+0.10775 \sin \left(\frac{3}{2} \theta\right) \\
0
\end{array}\right)
\end{gathered}
$$

and the dual shadow functions are:

$$
\begin{gathered}
\Psi_{0}^{\left(-\alpha_{2}\right)}(r, \theta)=0.05542 r^{-\frac{1}{2}}\left(\begin{array}{c}
\cos \left(\frac{1}{2} \theta\right)+4.6 \cos \left(\frac{3}{2} \theta\right) \\
\sin \left(\frac{1}{2} \theta\right)-2.6 \sin \left(\frac{3}{2} \theta\right) \\
0
\end{array}\right), \quad \Psi_{1}^{\left(-\alpha_{2}\right)}(r, \theta)=0.05542 r^{\frac{1}{2}}\left(\begin{array}{c}
0 \\
0 \\
-2 \cos \left(\frac{3}{2} \theta\right)
\end{array}\right) \\
\Psi_{2}^{\left(-\alpha_{2}\right)}(r, \theta)=0.05542 r^{\frac{3}{2}}\left(\begin{array}{c}
-0.27067 \cos \left(\frac{1}{2} \theta\right)-0.3 \cos \left(\frac{3}{2} \theta\right) \\
-0.31067 \sin \left(\frac{1}{2} \theta\right)+1.3 \sin \left(\frac{3}{2} \theta\right) \\
0
\end{array}\right)
\end{gathered}
$$



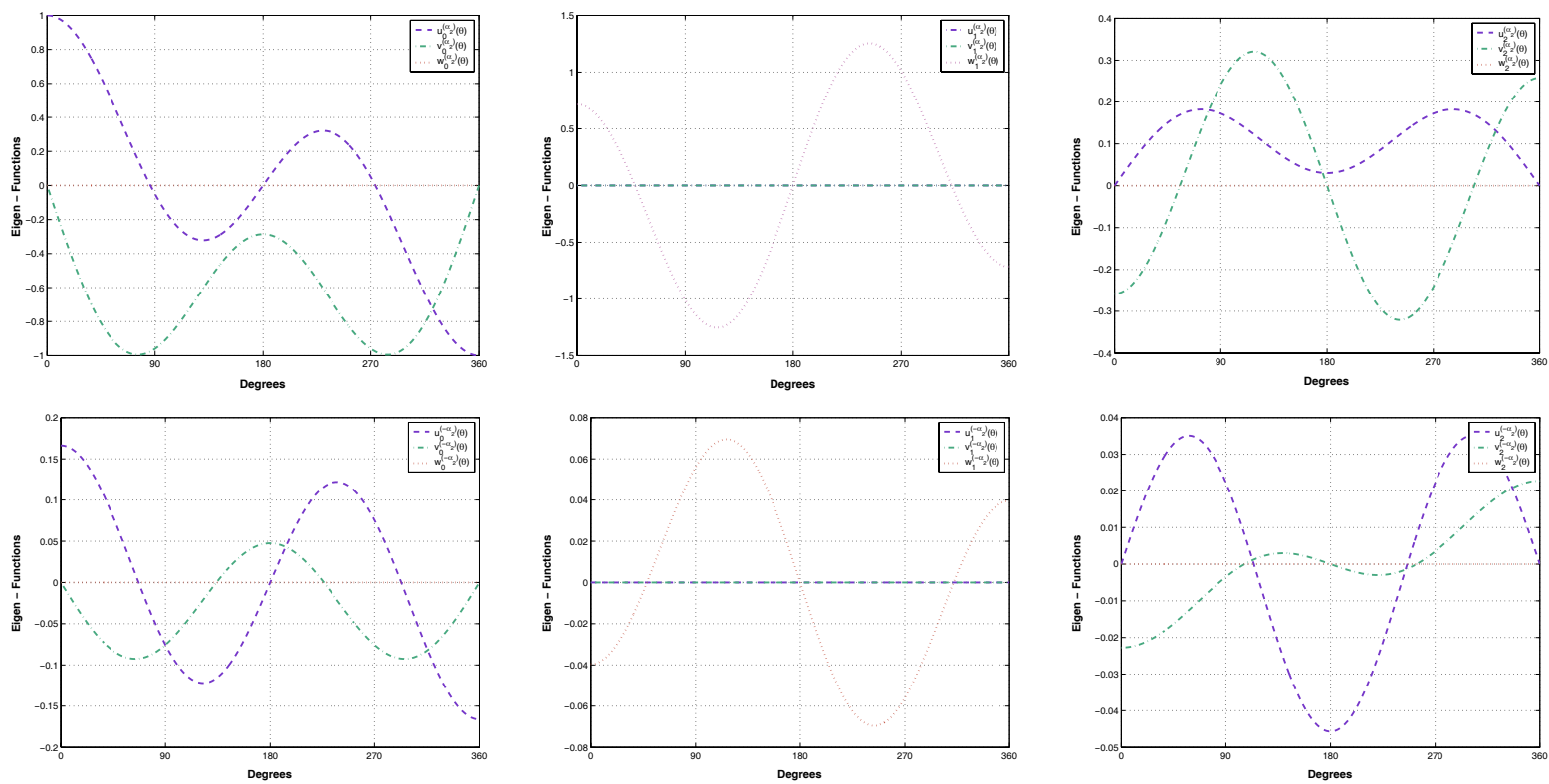

Figure 18. The eigen-functions (Top) and the dual eigen-functions (Bottom) associated with $\alpha_{2}=\frac{1}{2}$ in the case of cracked domain $(\omega=2 \pi), \lambda=0.5769$ and $\mu=0.3846$.

The primal and dual eigen- and shadow-functions associated with $\alpha_{2}=1 / 2$ are presented in figure 18 .

The third eigen-value in the case of cracked domain with traction free boundary conditions is $\alpha_{3}=1 / 2$ and the primal and shadow functions where $\lambda=0.5769$ and $\mu=0.3846$ are:

$$
\begin{gathered}
\boldsymbol{\Phi}_{0}^{\left(\alpha_{3}\right)}(r, \theta)=r^{\frac{1}{2}}\left(\begin{array}{c}
0 \\
0 \\
\cos \left(\frac{1}{2} \theta\right)
\end{array}\right), \quad \boldsymbol{\Phi}_{1}^{\left(\alpha_{3}\right)}(r, \theta)=r^{\frac{3}{2}}\left(\begin{array}{c}
-0.29333 \cos \left(\frac{1}{2} \theta\right) \\
0.10667 \sin \left(\frac{1}{2} \theta\right) \\
0
\end{array}\right) \\
\boldsymbol{\Phi}_{2}^{\left(\alpha_{3}\right)}(r, \theta)=r^{\frac{5}{2}}\left(\begin{array}{c}
0 \\
0 \\
-0.3 \cos \left(\frac{1}{2} \theta\right)
\end{array}\right)
\end{gathered}
$$

and the dual shadow functions are:

$$
\begin{gathered}
\Psi_{0}^{\left(-\alpha_{3}\right)}(r, \theta)=0.82760 r^{-\frac{1}{2}}\left(\begin{array}{c}
0 \\
0 \\
\cos \left(\frac{1}{2} \theta\right)
\end{array}\right), \quad \Psi_{1}^{\left(-\alpha_{3}\right)}(r, \theta)=0.82760 r^{\frac{1}{2}}\left(\begin{array}{c}
-0.13333 \cos \left(\frac{1}{2} \theta\right) \\
-0.53333 \sin \left(\frac{1}{2} \theta\right) \\
0
\end{array}\right) \\
\Psi_{2}^{\left(-\alpha_{3}\right)}(r, \theta)=0.82760 r^{\frac{3}{2}}\left(\begin{array}{c}
0 \\
0 \\
-1.16667 \cos \left(\frac{1}{2} \theta\right)
\end{array}\right)
\end{gathered}
$$

The primal and dual eigen- and shadow-functions associated with $\alpha_{3}=1 / 2$ are presented in figure 19 . 

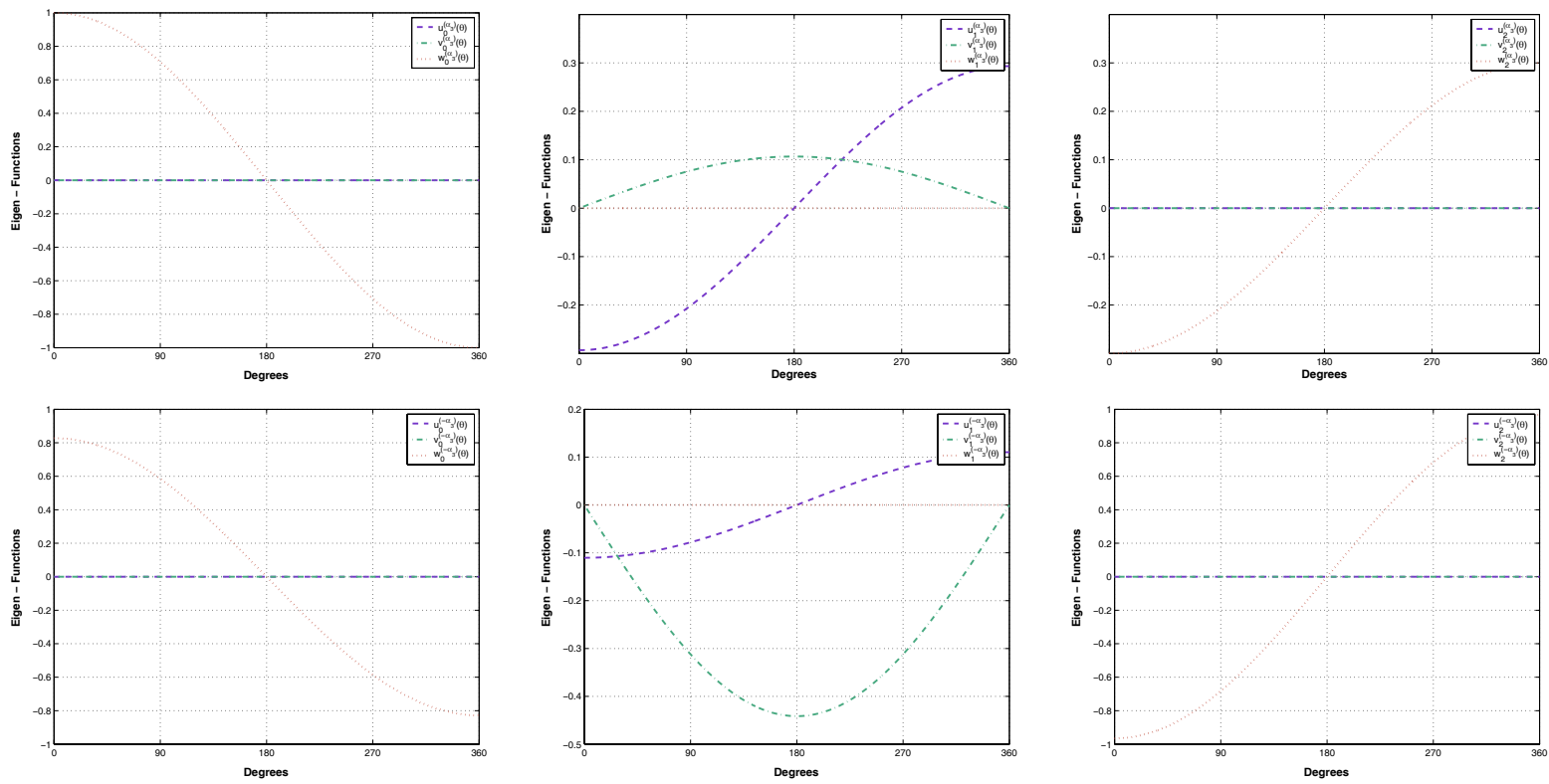

Figure 19. The eigen-functions (Top) and the dual eigen-functions (Bottom) associated with $\alpha_{3}=\frac{1}{2}$ in the case of cracked domain $(\omega=2 \pi), \lambda=0.5769$ and $\mu=0.3846$. 


\section{Appendix B}

\section{Primal and dual eigen- and shadow-functions for clamped $270^{\circ}$ V-notch.}

The displacements $\tilde{\boldsymbol{u}}((17))$ in the case of a V-notched domain $\left(\omega=\frac{3 \pi}{2}\right)$ with clamped boundary conditions on the surfaces $\Gamma_{1}$ and $\Gamma_{2}$ is constructed by the primal and shadow functions $\boldsymbol{\Phi}_{j}, j \geq 0$.

The primal and shadow functions $\boldsymbol{\Phi}_{0}, \boldsymbol{\Phi}_{1}, \boldsymbol{\Phi}_{2}$ as well as the dual shadow functions $\boldsymbol{\Psi}_{0}$, $\Psi_{1}, \Psi_{2}$ are the solutions of the differential equations in (11). The boundary conditions applied are prescribed in (25). There are an infinite number of eigen-values $\alpha_{i}$ for which there is an associated $\boldsymbol{\Phi}_{0}^{\left(\alpha_{i}\right)}$ and $\boldsymbol{\Psi}_{0}^{\left(\alpha_{i}\right)}$ where the positive $\alpha_{i}$ 's are associated with $\boldsymbol{\Phi}_{0}^{\left(\alpha_{i}\right)}$ and the negative $\alpha_{i}$ 's are associated with $\Psi_{0}^{\left(\alpha_{i}\right)}$. We consider the first three eigen-value only of the $270^{\circ} \mathrm{V}$ notched domain $\left(\omega=\frac{3 \pi}{2}\right), \alpha_{1}=0.595156, \alpha_{2}=0.759042, \alpha_{3}=0.66667$. The dual eigen function $\mathbf{\Psi}_{0}^{\left(\alpha_{i}\right)}$ includes the normalization factor $c_{0}^{\left(\alpha_{i}\right)}$, chosen such that the primal and dual eigen-functions satisfy the orthonormal condition as defined in (52).

The primal and shadow functions for a clamped $270^{\circ}$ V-notched domain associated with $\alpha_{1}=0.595156$ where $\lambda=0.5769$ and $\mu=0.3846$ are:

$$
\begin{aligned}
& \Phi_{0}^{\left(\alpha_{1}\right)}(r, \theta)=r^{0.59516}\left(\begin{array}{c}
-1.40993 \cos (0.40484 \theta)+1.40993 \cos (1.59516 \theta)+\sin (0.40484 \theta)-1.98793 \sin (1.59516 \theta) \\
1.98794 \cos (0.40484 \theta)-1.98794 \cos (1.59516 \theta)+2.80286 \sin (0.40484 \theta)-1.40993 \sin (1.59516 \theta) \\
0
\end{array}\right) \\
& \Phi_{1}^{\left(\alpha_{1}\right)}(r, \theta)=r^{1.59516}\left(\begin{array}{c}
0 \\
0 \\
1.17022 \cos (0.40484 \theta)-1.17022 \cos (1.59516 \theta)-0.82998 \sin (0.40484 \theta)+1.64996 \sin (1.59516 \theta)
\end{array}\right) \\
& \Phi_{2}^{\left(\alpha_{1}\right)}(r, \theta)=r^{2.59516}\left(\begin{array}{c}
-0.14583 \cos (0.40484 \theta)+0.14583 \cos (1.59516 \theta)+0.10343 \sin (0.40484 \theta)-0.20562 \sin (1.59516 \theta) \\
-0.31156 \cos (0.40484 \theta)+0.31156 \cos (1.59516 \theta)-0.43928 \sin (0.40484 \theta)+0.22097 \sin (1.59516 \theta) \\
0
\end{array}\right)
\end{aligned}
$$

and the dual shadow functions are:

$$
\begin{gathered}
\Psi_{0}^{\left(-\alpha_{1}\right)}(r, \theta)=-0.05898 r^{-0.59516}\left(\begin{array}{c}
0.70924 \cos (0.40484 \theta)-0.70924 \cos (1.59516 \theta)-0.50303 \sin (0.40484 \theta)+\sin (1.59516 \theta) \\
-0.50303 \cos (0.404844 \theta)+0.50303 \cos (1.59516 \theta)-0.70924 \sin (0.40484 \theta)+0.35677 \sin (1.59516 \theta) \\
0
\end{array}\right) \\
\Psi_{1}^{\left(-\alpha_{1}\right)}(r, \theta)=-0.05898 r^{0.40484}\left(\begin{array}{c}
0 \\
0 \\
-0.296125 \cos (0.40484 \theta)+0.29612 \cos (1.59516 \theta)+0.21002 \sin (0.40484 \theta)-0.41751 \sin (1.59516 \theta)
\end{array}\right) \\
\Psi_{2}^{\left(-\alpha_{1}\right)}(r, \theta)=-0.05898 r^{1.40484}\left(\begin{array}{c}
0.07044 \cos (0.40484 \theta)-0.07044 \cos (1.59516 \theta)+0.15980 \sin (0.40484 \theta)-0.11044 \sin (1.59516 \theta) \\
0.32312 \cos (0.40484 \theta)-0.32312 \cos (1.59516 \theta)+0.02033 \sin (0.40484 \theta)+0.20608 \sin (1.59516 \theta) \\
0
\end{array}\right)(60)
\end{gathered}
$$

The primal and dual eigen- and shadow-functions associated with $\alpha_{1}=0.595156$ are presented in Figure 20.

The primal and shadow functions $\boldsymbol{\Phi}_{0}^{\left(\alpha_{i}\right)}$ in the case of a clamped $270^{\circ}$ v-notched domain associated with $\alpha_{2}=0.759042$ where $\lambda=0.5769$ and $\mu=0.3846$ are: 

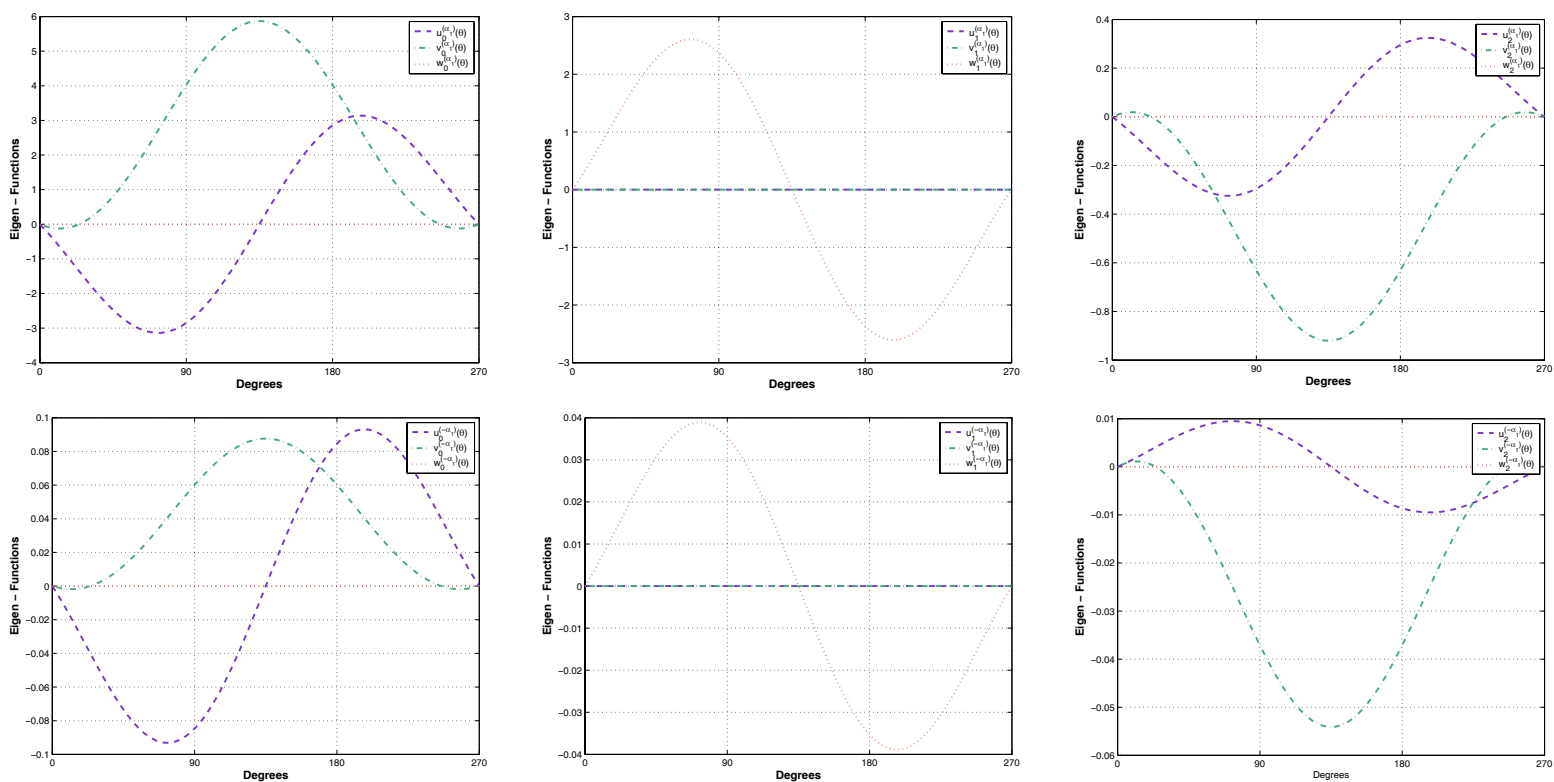

Figure 20. Eigen-functions (Top) and the dual eigen-functions (Bottom) associated with $\alpha_{1}=0.595156$ for a clamped V-notched domain $\left(\omega=\frac{3 \pi}{2}\right), \lambda=0.5769$ and $\mu=0.3846$.

$$
\begin{gathered}
\Phi_{0}^{\left(\alpha_{2}\right)}(r, \theta)=r^{0.75904}\left(\begin{array}{c}
1.56791 \cos (0.24096 \theta)-1.56791 \cos (1.75904 \theta)+\sin (0.24096 \theta)-2.45835 \sin (1.75904 \theta) \\
2.45835 \cos (0.24096 \theta)-2.45835 \cos (1.75904 \theta)-3.85448 \sin (0.24096 \theta)+1.56791 \sin (1.75904 \theta)
\end{array}\right) \\
0 \\
\Phi_{1}^{\left(\alpha_{2}\right)}(r, \theta)=r^{1.75904}\left(\begin{array}{c}
0 \\
0 \\
-1.50622 \cos (0.24096 \theta)+1.50622 \cos (1.75904 \theta)-0.96065 \sin (0.24096 \theta)+2.36163 \sin (1.75904 \theta)
\end{array}\right) \\
\Phi_{2}^{\left(\alpha_{2}\right)}(r, \theta)=r^{2.75904}\left(\begin{array}{c}
-0.15202 \cos (0.24096 \theta)+0.15202 \cos (1.75904 \theta)+0.10782 \sin (0.24096 \theta)+0.03358 \sin (1.75904 \theta) \\
-0.27837 \cos (0.24096 \theta)+0.27837 \cos (1.75904 \theta)-0.39248 \sin (0.24096 \theta)+0.65140 \sin (1.75904 \theta) \\
0
\end{array}\right)
\end{gathered}
$$

and the dual shadow functions are:

$$
\begin{aligned}
& \Psi_{0}^{\left(-\alpha_{2}\right)}(r, \theta)=-0.05520 r^{-0.75904}\left(\begin{array}{c}
-0.63779 \cos (0.240956 \theta)+0.63779 \cos (1.75904 \theta)-0.40678 \sin (0.24096 \theta)+\operatorname{Sin}(1.75904 \theta) \\
-0.40678 \cos (0.24096 \theta)+0.40678 \cos (1.75904 \theta)+0.63779 \sin (0.24096 \theta)-0.25944 \sin (1.75904 \theta)
\end{array}\right) \\
& 0 \\
& \Psi_{1}^{\left(-\alpha_{2}\right)}(r, \theta)=-0.05520 r^{0.24096}\left(\begin{array}{c}
0 \\
0 \\
0.24923 \cos (0.24096 \theta)-0.24923 \cos (1.75904 \theta)+0.15896 \sin (0.24096 \theta)-0.39077 \sin (1.75904 \theta)
\end{array}\right) \\
& \Psi_{2}^{\left(-\alpha_{2}\right)}(r, \theta)=-0.05520 r^{1.24096}\left(\begin{array}{c}
-0.00053 \cos (0.24096 \theta)+0.00053 \cos (1.75904 \theta)+0.00016 \sin (0.24096 \theta)+0.00034 \sin (1.75904 \theta) \\
0.17120 \cos (0.24096 \theta)-0.17120 \cos (1.75904 \theta)-0.42767 \sin (0.24096 \theta)+0.26843 \sin (1.75904 \theta) \\
0
\end{array}\right)(62)
\end{aligned}
$$

The primal and dual eigen- and shadow-functions associated with $\alpha_{2}=0.759042$ are presented in Figure 21.

The primal and shadow functions for a clamped $270^{\circ}$ v-notched domain associated with $\alpha_{3}=$ 0.666667 where $\lambda=0.5769$ and $\mu=0.3846$ are:

$$
\begin{gathered}
\boldsymbol{\Phi}_{0}^{\left(\alpha_{3}\right)}(r, \theta)=r^{0.66667}\left(\begin{array}{c}
0 \\
0 \\
\sin (0.66667 \theta)
\end{array}\right), \quad \boldsymbol{\Phi}_{1}^{\left(\alpha_{3}\right)}(r, \theta)=r^{1.66667}\left(\begin{array}{c}
-0.28846 \sin (0.66667 \theta) \\
0 \\
0
\end{array}\right) \\
\boldsymbol{\Phi}_{2}^{\left(\alpha_{3}\right)}(r, \theta)=r^{2.66667}\left(\begin{array}{c}
0 \\
0 \\
-0.23654 \sin (0.66667 \theta)
\end{array}\right)
\end{gathered}
$$



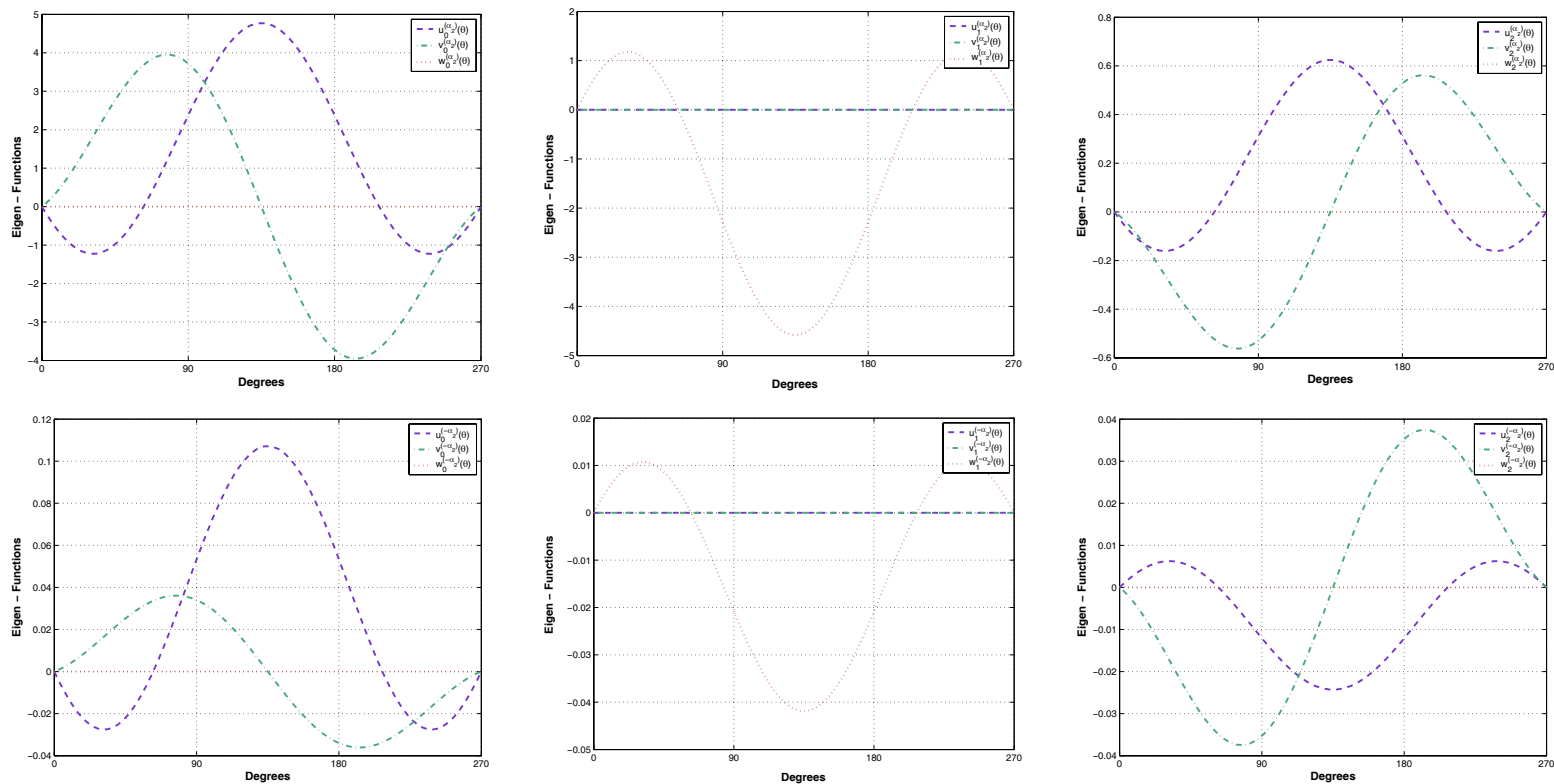

Figure 21. Eigen-functions (Top) and dual eigen-functions (Bottom) associated with $\alpha_{2}=0.759042$ for a clamped $V$-notched domain $\left(\omega=\frac{3 \pi}{2}\right), \lambda=0.5769$ and $\mu=0.3846$.

and the dual shadow functions are:

$$
\begin{gathered}
\Psi_{0}^{\left(\alpha_{3}\right)}(r, \theta)=-0.82760 r^{-0.66667}\left(\begin{array}{c}
0 \\
0 \\
-\sin (0.66667 \theta)
\end{array}\right), \quad \Psi_{1}^{\left(\alpha_{3}\right)}(r, \theta)=-0.82760 r^{0.33333}\left(\begin{array}{c}
0.46875 \sin (0.66667 \theta) \\
0 \\
0
\end{array}\right) \\
\Psi_{2}^{\left(\alpha_{3}\right)}(r, \theta)=-0.82760 r^{1.33333}\left(\begin{array}{c}
0 \\
0 \\
1.45313 \sin (0.66667 \theta)
\end{array}\right)
\end{gathered}
$$

The primal and dual eigen- and shadow-functions associated with $\alpha_{3}=0.666667$ are presented in Figure 22.

\section{Appendix $\mathrm{C}$}

\section{Why $B J_{2}$ is insufficient for extraction purposes when $K_{2}$ is used?}

Although the extraction polynomials $B J_{2}^{(k)}$ satisfy the conditions in (29) when using the dual singular functions $K_{0}^{\left(\alpha_{i}\right)}, K_{1}^{\left(\alpha_{i}\right)}$ and $K_{2}^{\left(\alpha_{i}\right)}$, we selected $B J_{4}^{(k)}$ in order to extract the ESIF's (see subsection 3.2 ). In this section we examine the hierarchic family $B J_{2}^{(k)}$ and present the reasons for preferring $B J_{4}^{(k)}$.

\section{C.1. Jacobi Extraction Polynomials of Order 2}

The Jacobi Polynomials of Order 2 are of the form (see [2, pp. 773-774]):

$$
J_{2}^{(k)}\left(x_{3}\right)=\frac{1}{k^{2}+7 k+12} \sum_{l=0}^{k} \frac{(k+l+4) !}{2^{l} l !(k-l) !(2+l) !}\left(x_{3}-1\right)^{l}
$$



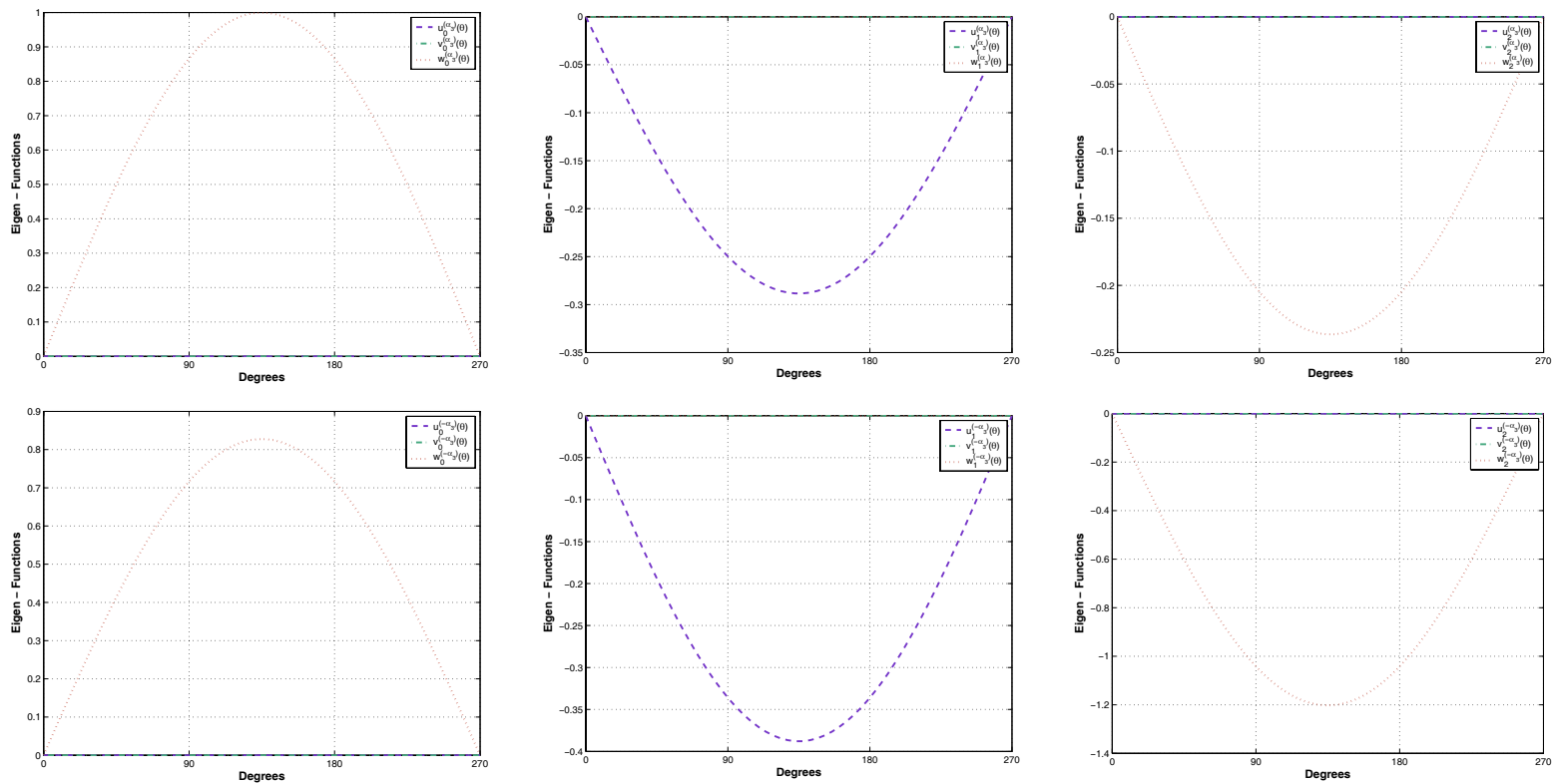

Figure 22. Eigen-functions (Top) and dual eigen-functions (Bottom) associated with $\alpha_{3}=0.666667$ for a clamped $V$-notched domain $\left(\omega=\frac{3 \pi}{2}\right), \lambda=0.5769$ and $\mu=0.3846$.

and the constant $h_{k}$ in $(32)$ is

$$
h_{k}=\frac{2^{5}(k+1)(k+2)}{(2 k+5)(k+3)(k+4)}
$$

Inserting (66) and (65) in (33), we finally obtain:

$$
B J_{2}^{(k)}\left(x_{3}\right)=\frac{(2 k+5)(k+3)(k+4)}{2^{5}(k+1)(k+2)} \frac{\left(1-x_{3}^{2}\right)^{2}}{k^{2}+7 k+12} \sum_{l=0}^{k} \frac{(k+l+4) !}{2^{l} l !(k-l) !(2+l) !}\left(x_{3}-1\right)^{l} .
$$

The computation of $J[R]$ using $B J_{2}^{(k)}$ requires the value of $B J_{2}^{(k)}, \partial_{3} B J_{2}^{(k)}, \partial_{3}^{2} B J_{2}^{(k)}$ and $\partial_{3}^{3} B J_{2}^{(k)}$ at the Gauss quadrate points, along the $x_{3}$ axis. The polynomials $B J_{2}^{(k)},(0 \leq k \leq 5)$, and their first three derivatives are presented in figure 23.

One may see that $\left.B J_{2}^{(k)}\right|_{x_{3}= \pm 1}=\left.\partial_{3} B J_{2}^{(k)}\right|_{x_{3}= \pm 1}=0$, as expected. The derivatives $\partial_{3}^{2} B J_{2}^{(k)}$ and $\partial_{3}^{3} B J_{2}^{(k)}$ however have large gradients in the vicinity of $x_{3}= \pm 1$. In fact, as $k$ increases, the second and third derivatives of $B J_{2}^{(k)}$ have larger gradients at the boundaries $x_{3}= \pm 1$.

\section{C.2. ESIF Extraction USING $B J_{2}^{(k)}$}

To examine the influence of these large gradients we compute $J[R]$ at different values of $R$ using a Gauss quadrature of order 10 in both $\theta$ and $x_{3}$ directions and with $p=7$ in finite element analysis.

We plot in figure $24 \log \left(\left(J_{\mathrm{ex}}-J[R]\right) / J_{\mathrm{ex}}\right)$ vis. $\log (R)$, showing the numerical convergence rate. $J[R]$ is computed using $B J_{2}^{(0)}\left(x_{3}\right), B J_{2}^{(1)}\left(x_{3}\right), B J_{2}^{(2)}\left(x_{3}\right)$ and $K_{2}^{\left(\alpha_{1}\right)}$. The results of $J[R]$ are summarized in Table II. 

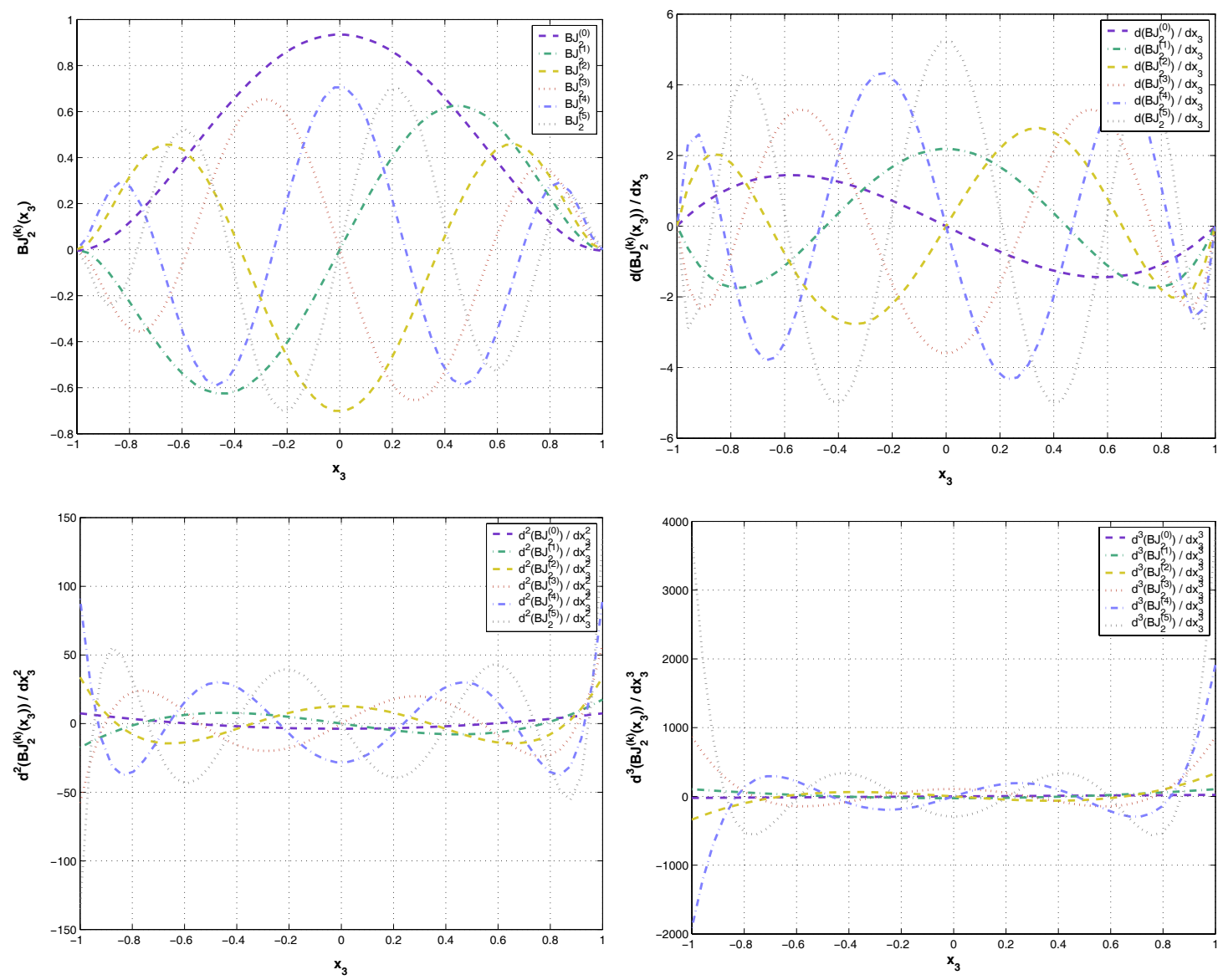

Figure 23. $B J_{2}^{(k)}\left(x_{3}\right)$ and the derivatives $\partial_{3} B J_{2}^{(k)}\left(x_{3}\right), \partial_{3}^{2} B J_{2}^{(k)}\left(x_{3}\right)$ and $\partial_{3}^{3} B J_{2}^{(k)}\left(x_{3}\right)$ where $0 \leq k \leq 5$

We can notice in Figure 24 that the convergence rate is at least of order $R^{3}$, as expected, but the relative error is still large even at $R=0.05$ as can be observed in Table II. The large error is especially expressed at the results obtained for the third eigen-value, $\alpha_{3}$. It is easy to see that the results of $J[R]$ obtained by using $B J_{4}^{(k)}$ (as presented in Table I) as much more accurate than the results obtained by using $B J_{2}^{(k)}$ (as presented in Table II).

Because the results obtained using $B J_{2}^{(k)}$ are as expected by the theory, we extracted the ESIF of order $2,3,4,5$ and computed its relative error using the data at $R=0.05$. The exact ESIF's are $A_{1}=3+4 x_{3}+5 x_{3}^{2}, A_{2}=2+3 x_{3}+4 x_{3}^{2}$ and $A_{3}=5+4 x_{3}+2 x_{3}^{2}$. The relative error obtained is presented in Figure 25.

As seen in Figure 25, as we increase the order of the ESIF, the relative error in the vicinity of $x_{3}=-1$ and $x_{3}=1$ increases. This result is obtained due to the large gradients of the second and third derivatives of $B J_{2}^{(k)}$ close to $x_{3}= \pm 1$ - see also this phenomenon in the results presented in [12]. This phenomenon does not obtained once computing the ESIF using $B J_{4}^{(k)}$ as can be observed in Figure 6 .

The large gradients of the ESIF is more pronounced in the results of the third eigen-value, $\alpha_{3}$. Therefore, we extracted $A_{3}\left(x_{3}\right)$ of order 5 at different $R$ 's, and extrapolated the ESIF to 


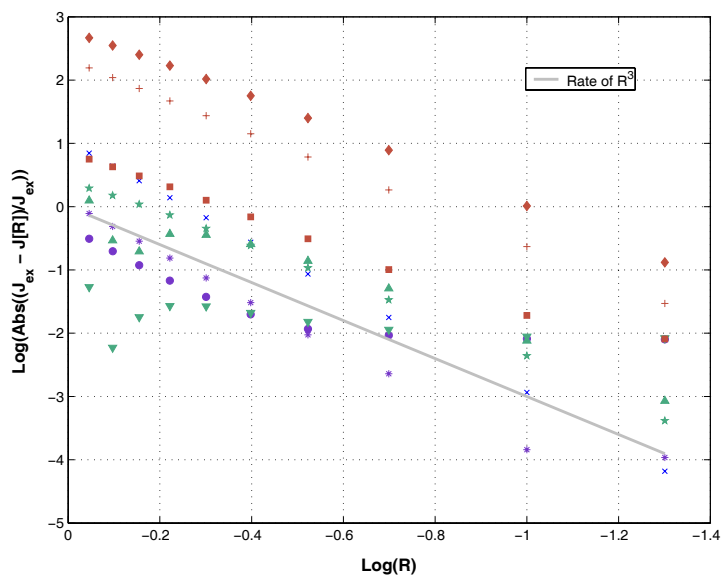

- $\tilde{a}_{0}^{\left(\alpha_{1}\right)}$

* $\tilde{a}_{1}^{\left(\alpha_{1}\right)}$

$\times \tilde{a}_{2}^{\left(\alpha_{1}\right)}$

$\nabla \tilde{a}_{0}^{\left(\alpha_{2}\right)}$

$\star \tilde{a}_{1}^{\left(\alpha_{2}\right)}$

$\Delta \tilde{a}_{2}^{\left(\alpha_{2}\right)}$

- $\tilde{a}_{0}^{\left(\alpha_{3}\right)}$

$+\tilde{a}_{1}^{\left(\alpha_{3}\right)}$

$\wedge \tilde{a}_{2}^{\left(\alpha_{3}\right)}$

Figure 24. Convergence rate of $J[R]$ using $B J_{2}^{(k)}, k=0,1,2$ and $K_{2}^{\left(\alpha_{i}\right)}$ for cracked domain with traction free boundary conditions, $\lambda=0.5769$ and $\mu=0.3846$.
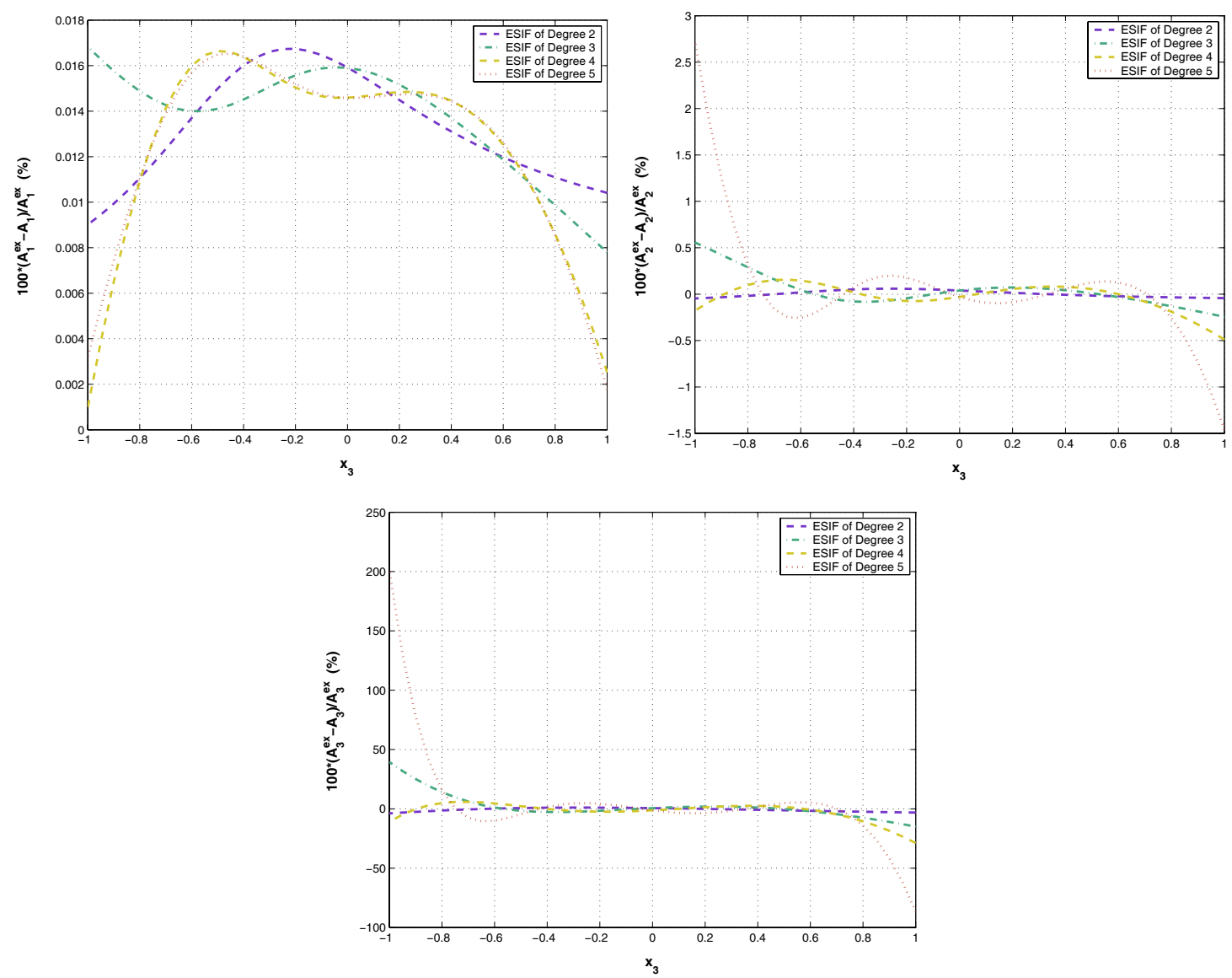

Figure 25. Relative error (\%) of the extracted ESIF using $B J_{2}^{(k)}, k \leq 5$ for cracked domain with traction free boundary conditions, $\lambda=0.5769$ and $\mu=0.3846 . A_{1}^{e x}\left(x_{3}\right)=3+4 x_{3}+5 x_{3}^{2}, A_{2}^{e x}\left(x_{3}\right)=2+3 x_{3}+4 x_{3}^{2}$, $A_{3}^{e x}\left(x_{3}\right)=5+4 x_{3}+2 x_{3}^{2}$

$R=0$ using the values obtained at $R=0.8,0.2,0.05$, knowing that the error behaves as $\mathcal{O}\left(R^{3}\right)$. The results are presented in Figure 26. The accuracy of the results obtained by Richardson extrapolation is higher even of the accuracy of the result obtained using $R=0.05$. We conclude 
Table II. Numerical results of $J[R]$ using $B J_{2}^{(k)}, k=3,4,5$ and $K_{2}^{\left(\alpha_{i}\right)}$, for cracked domain with traction free boundary conditions, $\lambda=0.5769$ and $\mu=0.3846$.

\begin{tabular}{|c|c|c|c|c|c|c|}
\hline & \multicolumn{6}{|c|}{$\alpha_{1}=0.5$} \\
\hline$R$ & $J_{e x}$ & $B J_{2}^{(3)}$ & $J_{e x}$ & $B J_{2}^{(4)}$ & $J_{e x}$ & $B J_{2}^{(5)}$ \\
\hline 0.9 & 0 & 3.42799 & 0 & 13.50506 & 0 & 8.01406 \\
\hline 0.8 & 0 & 2.14041 & 0 & 8.43103 & 0 & 5.00271 \\
\hline 0.7 & 0 & 1.25450 & 0 & 4.94224 & 0 & 2.93283 \\
\hline 0.6 & 0 & 0.67705 & 0 & 2.66751 & 0 & 1.58312 \\
\hline 0.5 & 0 & 0.32661 & 0 & 1.28634 & 0 & 0.76332 \\
\hline 0.4 & 0 & 0.13383 & 0 & 0.52706 & 0 & 0.31268 \\
\hline 0.3 & 0 & 0.04228 & 0 & 0.16668 & 0 & 0.09895 \\
\hline 0.2 & 0 & 0.00840 & 0 & 0.03286 & 0 & 0.01951 \\
\hline 0.1 & 0 & $5.12 \mathrm{E}-04$ & 0 & 0.00191 & 0 & 0.00114 \\
\hline 0.05 & 0 & $3.13 \mathrm{E}-05$ & 0 & $4.23 \mathrm{E}-05$ & 0 & $4.06 \mathrm{E}-06$ \\
\hline
\end{tabular}

\begin{tabular}{|c|c|c|c|c|c|c|c|c|c|c|c|c|}
\hline \multirow[b]{2}{*}{$R$} & \multicolumn{6}{|c|}{$\alpha_{2}=0.5$} & \multicolumn{6}{|c|}{$\alpha_{3}=0.5$} \\
\hline & $J_{e x}$ & $B J_{2}^{(3)}$ & $J_{e x}$ & $B J_{2}^{(4)}$ & $J_{e x}$ & $B J_{2}^{(5)}$ & $\| J_{e x}$ & $B J_{2}^{(3)}$ & $\| J_{e x}$ & $B J_{2}^{(4)}$ & $\| J_{e x}$ & $B J_{2}^{(5)}$ \\
\hline 0.9 & 0 & 6.45270 & 0 & -1.92391 & 0 & 15.08370 & 0 & 684.89057 & 0 & 358.50386 & 0 & 1601.03859 \\
\hline 0.8 & 0 & 4.96463 & 0 & -0.45001 & 0 & 11.60613 & 0 & 482.19792 & 0 & 270.91136 & 0 & 1127.22146 \\
\hline 0.7 & 0 & 3.59874 & 0 & 0.30165 & 0 & 8.41273 & 0 & 324.69828 & 0 & 194.30498 & 0 & 759.03606 \\
\hline 0.6 & 0 & 2.42743 & 0 & 0.56981 & 0 & 5.67423 & 0 & 206.08012 & 0 & 130.44288 & 0 & 481.73890 \\
\hline 0.5 & 0 & 1.49162 & 0 & 0.54976 & 0 & 3.48683 & 0 & 120.49220 & 0 & 80.15835 & 0 & 281.66752 \\
\hline 0.4 & 0 & 0.80492 & 0 & 0.39421 & 0 & 1.88171 & 0 & 62.47922 & 0 & 43.42907 & 0 & 146.05863 \\
\hline 0.3 & 0 & 0.35565 & 0 & 0.21371 & 0 & 0.83134 & 0 & 26.76345 & 0 & 19.33200 & 0 & 62.56450 \\
\hline 0.2 & 0 & 0.10976 & 0 & 0.07734 & 0 & 0.25650 & 0 & 8.06996 & 0 & 6.02719 & 0 & 18.86400 \\
\hline 0.1 & 0 & 0.01421 & 0 & 0.01130 & 0 & 0.03314 & 0 & 1.02870 & 0 & 0.79061 & 0 & 2.40439 \\
\hline 0.05 & 0 & 0.00181 & 0 & 0.00147 & 0 & 0.00416 & 0 & 0.13001 & 0 & 0.10112 & 0 & 0.30372 \\
\hline
\end{tabular}
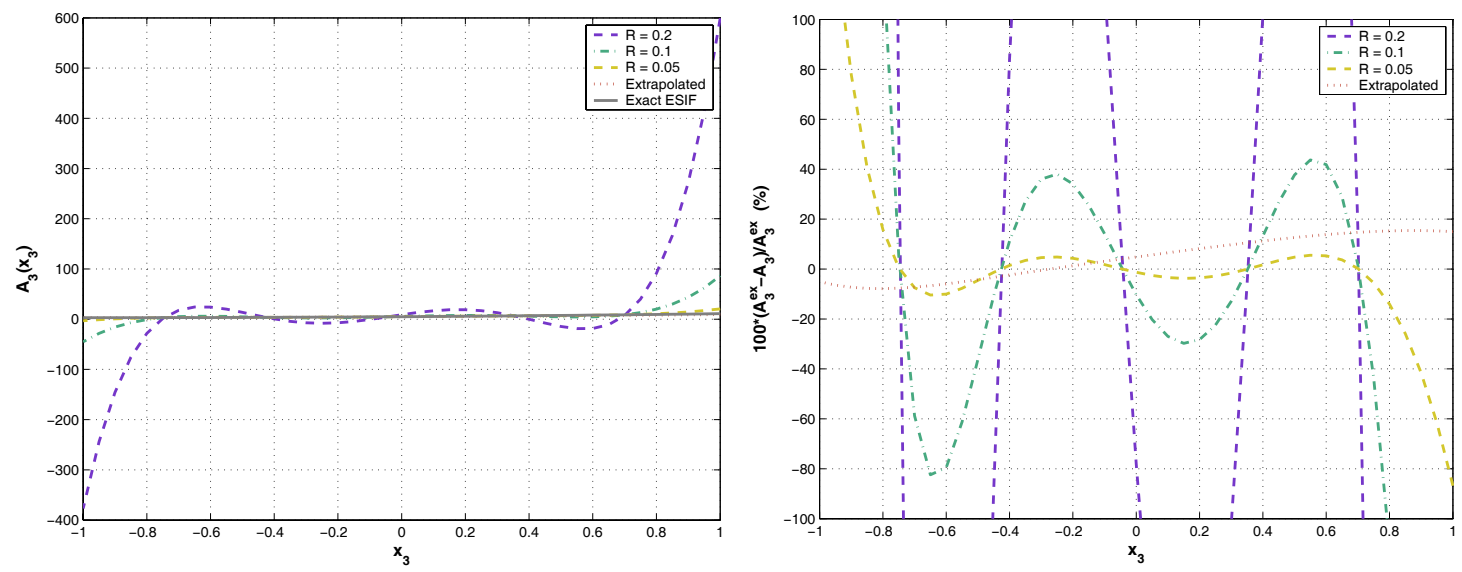

Figure 26. $A_{3}\left(x_{3}\right)$ (left) and its relative error (right) at $R=0.2, R=0.1, R=0.05$ and extrapolated to $R=0$ using $B J_{2}^{(k)}, k \leq 5$, where $A_{3}^{e x}\left(x_{3}\right)=5+4 x_{3}+2 x_{3}^{2}, \omega=2 \pi, \lambda=0.5769$ and $\mu=0.3846$.

at this point that convergence rate of $J[R]$ integral using the hierarchical family of polynomials $B J_{2}^{(k)}$ is as expected by the theory. However, because of large gradients of the second and third derivatives of the extraction polynomials $B J_{2}^{(k)}$, the accuracy of the ESIF obtained close to the singular point $\left(R=0.05\right.$ ) is not sufficient and contains large gradients in the vicinity of $x_{3}= \pm 1$. 
In order to avoid these large gradients resulting in poor accuracy of the computed ESIF, a better family of extraction polynomials is considered that have to to satisfy the condition in (29) and their derivatives have to be smoother than the derivatives of $B J_{2}^{(k)}$.

\section{C.3. Extraction Polynomials of Order 4}

If one chooses $B J_{4}^{(k)}(39)$ as the extraction polynomials, it satisfies the condition in (29) up to $m=4$, i.e.:

$$
\left.B J_{4}^{(k)}\right|_{x_{3}= \pm 1}=\left.\partial_{3} B J_{4}^{(k)}\right|_{x_{3}= \pm 1}=\left.\partial_{3}^{2} B J_{4}^{(k)}\right|_{x_{3}= \pm 1}=\left.\partial_{3}^{3} B J_{4}^{(k)}\right|_{x_{3}= \pm 1}=0
$$

The polynomials $B J_{4}^{(k)},(0 \leq k \leq 5)$, and their first three derivatives are presented in Figure 27 . Due to the extra two conditions that $B J_{4}^{(k)}$ satisfy $\left(\left.\partial_{3}^{2} B J_{4}^{(k)}\right|_{x_{3}= \pm 1}=\left.\partial_{3}^{3} B J_{4}^{(k)}\right|_{x_{3}= \pm 1}=0\right)$, there
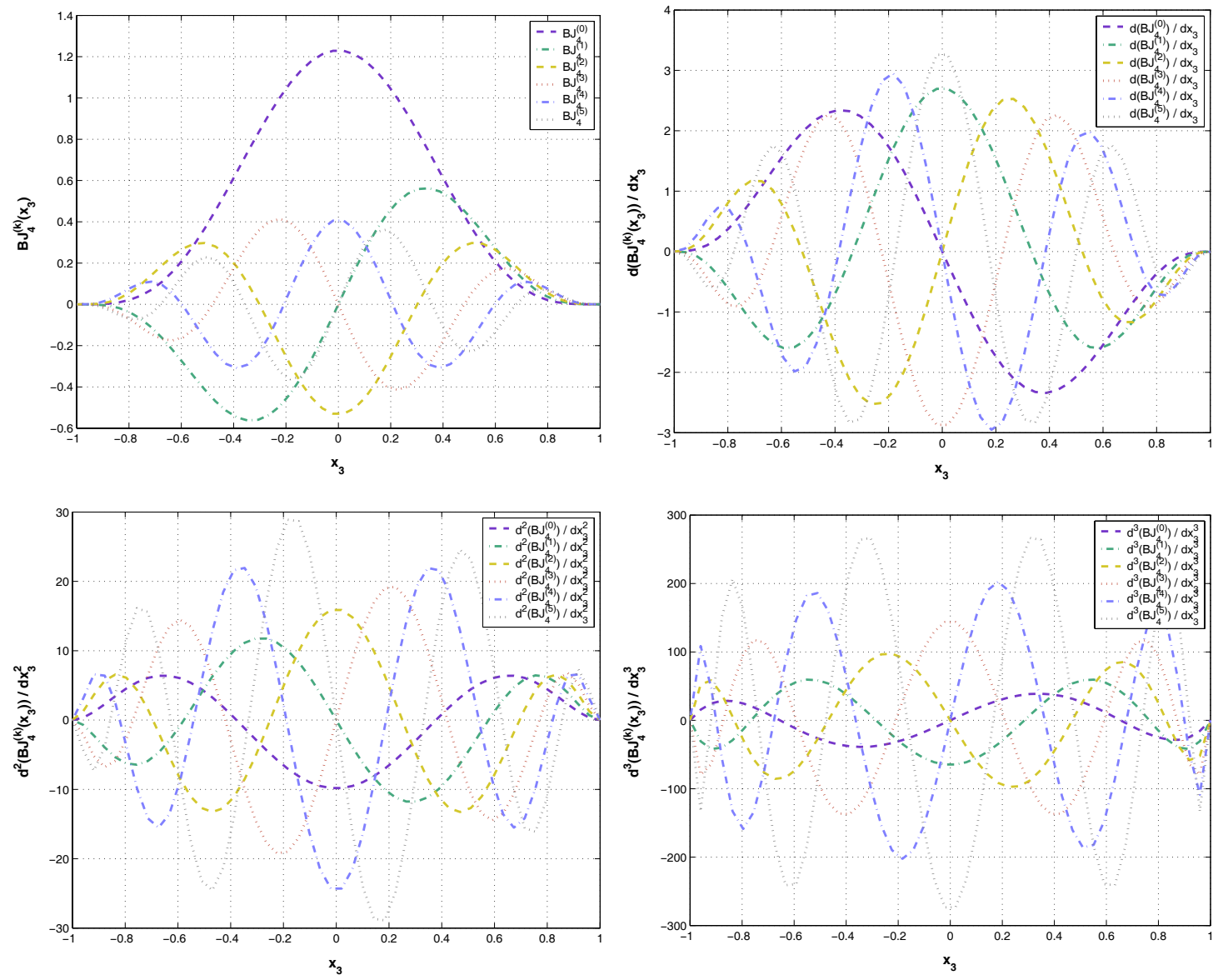

Figure 27. $B J_{4}^{(k)}\left(x_{3}\right)$ and the derivatives $\partial_{3} B J_{4}^{(k)}\left(x_{3}\right), \partial_{3}^{2} B J_{4}^{(k)}\left(x_{3}\right)$ and $\partial_{3}^{3} B J_{4}^{(k)}\left(x_{3}\right)$ where $0 \leq k \leq 5$

are no large gradients in the second and third derivatives of the polynomials and therefore the extracted ESIF at sufficiently small $R$ is of high accuracy, as presented in section 4 and section 5. 


\section{References}

1. STRESS CHECK Mster Guide - V-7. Engineering Software Research and Development, Inc., 7750 Clayton Road, Suite 204, St. Louis, MO 63117, www.esrd.com, 2004.

2. M. Abramowitz and A. Stegun. Handbook of mathematical functions with formulas, graphs and mathematical tables. Nat. Bureau of Standards, Applied Mathematics Series, 1964.

3. A. Beagles and A.-M. Sändig. Singularities of rotationally symmetric solutions of boundary value problems for the Lamé equations. ZAMM - Z. Angew. Math. Mech., 71:423-431, 1991.

4. M. Costabel, M. Dauge, and Y. Lafranche. Fast semi-analytic computation of elastic edge singularities. Computer Meth. Appl. Mech. Engrg., 190:2111-2134, 2001.

5. M. Costabel, M. Dauge, and Z. Yosibash. A quasidual function method for extracting edge stress intensity functions. SIAM Jour. Math. Anal., 35(5):1177-1202, 2004.

6. M. Dauge. Elliptic boundary value problems in corner domains - smoothness and asymptotics of solutions. Lecture notes in Mathematics 1341, Springer-Verlag, Heidelberg, 1988.

7. P. Grisvard. Elliptic problems in nonsmooth domains. Pitman Publishing, England, 1985.

8. R.J. Hartranft and G.C. Sih. The use of eigenfunction expansions in the general solution of three-dimensional crack problems. Jour. Math. Mech., 19(2):123-138, 1967.

9. M. F. Kanninen and C. H. Popelar. Advanced Fracture Mechanics. Oxford University Press, New-York, NY, USA, 1985.

10. S.N. Karp and F.C. Jr. Karal. The elastic field behavior in the neighborhood of a crack of arbitraty angle. Comm. on Pure \&s Appl. Math., 15:413-421, 1962.

11. D. Leguillon and E. Sanchez-Palencia. Computation of singular solutions in elliptic problems and elasticity. John Wiley \& Sons, New York, NY, 1987.

12. N. Omer, Z. Yosibash, M. Costabel, and M. Dauge. Edge flux intensity functions in polyhedral domains and their extraction by a quasidual function method. Int. Jour. Fracture, 129:97-130, 2004.

13. B. A. Szabó and Z. Yosibash. Numerical analysis of singularities in two-dimensions. Part 2: Computation of the generalized flux/stress intensity factors. Int. Jour. Numer. Meth. Engrg., 39(3):409-434, 1996.

14. M. L. Williams. Stress singularities resulting from various boundary conditions in angular corners of plates in extension. Trans. ASME, Jour. Appl. Mech., 19:526-528, 1952.

15. Z. Yosibash and B. A. Szabó. Numerical analysis of singularities in two-dimensions. Part 1: Computation of eigenpairs. Int. Jour. Numer. Meth. Engrg., 38(12):2055-2082, 1995. 